\title{
Modal analysis of spindles while accounting for system decay and its application to machine tool chatter prevention
}

\author{
Omar Gaber • Seyed M. Hashemi
}

Received: 24 October 2014 / Accepted: 26 February 2015 / Published online: 24 March 2015

(C) Springer-Verlag London 2015

\begin{abstract}
This paper investigates the vibrational characteristics of a machining spindle over its life span. The experimental investigation was carried out using tap testing, where the fundamental frequencies of the spindle system were recorded for different spindle categories, namely, 'production' and 'proveout' spindles. Focussing on production spindles, the system ageing translated through a reduction in the system's natural frequency is modelled as changes in the bearings' stiffness. The experimentally evaluated natural frequencies were then used to calculate the equivalent bearings' stiffness within the spindle by means of a calibrated dynamic stiffness method (CDSM) at various stages of spindle's life. A comparison between the stability lobes generated for two different instances in time, in a full slotting cuts process, shows that over the life span of a spindle, the stability lobes would shift sufficiently to cause chatter after initially being stable. Therefore, as the spindle ages, the presented methodology can be exploited to predict the updated machining parameters necessary to avoid unstable chatter conditions.
\end{abstract}

Keywords Spindle $\cdot$ Stability lobes $\cdot$ Dynamic stiffness method (DSM) · Natural frequency $\cdot$ Updated machining parameters

\section{Nomenclature \\ Abbreviations \\ 2D \\ $3 \mathrm{D}$ \\ $\mathrm{BC}$ \\ Two-dimensional \\ Three-dimensional \\ Boundary condition}

O. Gaber $(\bowtie) \cdot$ S. M. Hashemi

The Department of Aerospace Engineering, Ryerson University,

350 Victoria Street, Toronto, ON, Canada M5B2K3

e-mail: ogaber@ryerson.ca

S. M. Hashemi

e-mail: smhashem@ryerson.ca

$\begin{array}{ll}\text { CDSM } & \text { Calibrated dynamic stiffness matrix } \\ \text { CNC } & \text { Computer numerical control } \\ \text { DOF } & \text { Degree of freedom } \\ \text { DSM } & \text { Dynamic stiffness matrix } \\ \text { FEM } & \text { Finite element method } \\ \text { FRF } & \text { Frequency response function } \\ \text { RPM } & \text { Rounds per minute } \\ D=\mathrm{d} / \mathrm{d} \xi & \text { Differentiation operator } \\ \mathbf{F} & \text { Load vector } \\ K & \text { Coupled bending-torsion stiffness } \\ {[\boldsymbol{K}]} & \text { Static stiffness matrix } \\ {[\boldsymbol{M}]} & \text { Static mass matrix } \\ T & \text { Kinetic energy } \\ u & \text { In-plane bending displacement, } \\ U & \text { along } x \text {-axis } \\ v & \text { Potential energy } \\ v & \text { Out-of plane bending displacement, } \\ & \text { along } y \text {-axis }\end{array}$

Greek symbols

$\delta \quad$ Variational operator

$\delta \quad$ Displacement vector/vector of

degree-of-freedom/natural shape

$\varphi \quad$ Torsional twist

$\omega \quad$ Rotary frequency of oscillations

$\bar{\omega} \quad$ Average frequency value

$\omega_{\mathrm{n}} \quad$ Natural frequency

$\Omega \quad$ Rotational/rotational speed

$\xi=z / L \quad$ Non-dimensional length

\section{Superscripts/subscripts \\ $K_{\text {theo }} \quad$ Theoretical tool stiffness \\ $K_{\mathrm{S}} \quad$ Bearing's equivalent linear spring stiffness (constant) \\ $K^{\mathrm{e}}(\omega) \quad$ DSM of a beam element \\ $M_{x} \quad$ Bending moment about ' $x$ ' axis}


$M_{y} \quad$ Bending moment about ' $y$ ' axis

$S_{x} \quad$ Shear force along ' $x$ ' direction

$S_{y} \quad$ Shear force along ' $y$ ' direction

\section{Introduction}

Machining efficiency has been the main focus of many manufacturing firms for the past 50 years. As competition tightens, companies started to look for more effective ways to produce as many parts as possible in as little time as possible with minimal to no chatter. It has been well established that chatter is related to the overall spindle stiffness $[1,2]$. There have been numerous research studies dedicated to predict the machine spindle stiffness and, in turn, the bearing stiffness. Lin et al. [3] summarised the multitude of dynamic models of spindle and bearings found in the literature. Guo and Parker [4] investigated the effect of different assumptions being made in the literature on the bearing stiffness. They also studied the effect of load on the overall bearing stiffness. Numerically replacing bearings with springs has become common practice in modelling spindles. Zhu et al. [5] used this bearing/spring replacement to investigate its effect on the natural frequencies of the spindle. The position of the bearing was also investigated. Cao and Altintas [6] also modelled a full spindle and replaced the bearings with springs, where they also investigated the effect of preloading the bearing on the natural frequency of the spindle through varying the stiffness of the spring equivalent. Hajikolaei et al. [7] attempted the use of spindle speed variation and adaptive force regulation to suppress regenerative chatter in the turning process. It was found that both methods reduce chatter but varying spindle speed reduces chatter faster. Traditional chatter theory assumes that all cutting parameters stay constant. Graham et al. [8] took into consideration the variation in these parameters and observed their effects on stability lobes. Variation in tool tip dynamics and cutting force coefficient were considered. Using these variations, a stability range was suggested. Using a $2 \mathrm{D}$ finite element model, and taking the residual deposition stress and machine parameters into consideration, Qin et al. [9] also investigated the effects of tip geometry on the performance of diamond-coated cutting tools. It was found that there is a correlation between residual stresses, tool geometry, and tool performance.

The aim of the present paper is to examine the changes of spindle natural frequency caused by ageing (i.e. use and wear), and to present a systematic procedure for prediction of the optimal ranges of machining parameters required to maintain stable cutting conditions (as the spindle ages). The effectiveness and practical applicability of the proposed methodology is demonstrated through a real spindle example, made available to the authors for experimental tests. The system's fundamental frequencies are first evaluated at various stages of spindle's life, both experimentally and analytically by developing a calibrated dynamic stiffness method (CDSM). The CDSM model is outfitted with linear spring elements, where the spring constants are adjusted to reflect the spindle ageing translated into reduced bearings stiffness. Based on the resulting bearings stiffness values, the CDSM model of the spindle is then exploited to evaluate equivalent milling stiffness, which is, in turn, used to generate stability lobes for machining spindles as they age. As it will be shown, as the spindle ages and the bearings wear, the overall natural frequency of the system decreases and the stability lobes shift. A shift in the stability lobes could potentially move some cutting parameters from the stable to the unstable regions. To the best of authors' knowledge, such a systematic procedure to predict the updated machining parameters, necessary for stable cutting conditions, has not been reported in the open literature. Furthermore, while a DSM for the vibration analysis of simple (one-segment), uniform, spinning beams has been previously developed by Banerjee and Su [10], a CDSM method for the modelling of entire spindle systems, and its application to the chatter analysis and prevention, has not been yet used by researchers. As it will be further discussed later in the paper, the DSM formulation is based on the closed form solution to the governing equations of motion, exact within the limits of the theory [10]. In addition, the displacement field within each DSM element is assumed to be continuous, representing infinite number of degrees of freedom (DOFs) [10], while in the conventional FEM formulation, each element represents a limited number of nodal DOFs. Therefore, contrary to the conventional finite element methods (FEMs), each uniform segment of the spindle system can be advantageously modelled as a single DSM element, resulting in a much simpler model. Furthermore, a one-element per segment DSM model can result in infinite number of natural frequencies, exact within the limits of the theory, while the mesh in a conventional FEM model must be further refined, should one need to evaluate higher frequencies [10]. It is also worth noting that, in the present study, the case of a non-spinning spindle $(\Omega=0)$ is investigated, as the experimental modal analyses have been carried out on a stationary machine spindle. As a result, centrifugal and gyroscopic effects resulting from the spinning speed, already investigated, both analytically and numerically, in an earlier work by the authors [11], have not been considered in the analyses.

The present paper is divided in five sections. First, the development differential equations governing the bendingbending-torsion vibrations of a uniform spinning beam, exhibiting material couplings, followed by the corresponding DSM formulation, is briefly discussed. Applied to a typical spindle system, the DSM method is then validated against conventional FEM models, where the bearings are represented 
as simply supported boundary conditions (BC). A CDSM Model of the spindle system is then developed, where the simply supported BC are replaced with linear springs, to incorporate the inherent bearings flexibility into the model, and the stiffness values are determined through a comparison between the frequencies obtained using the DSM and experimental modal analyses. Next, the calculation of the so-called theoretical tool stiffness is briefly discussed. It is followed by the development of stability lobes for a machining process (aluminium) with a full slot axial depth of cut, and using a two-tooth roughing tool, based on the resulting system parameters. The system stability is also investigated experimentally to validate the theoretical method and code written to evaluate the stability lobes. Finally, the shift in the stability lobes caused by the spindle ageing (i.e. reduced frequencies) is discussed, and it is shown that the updated machining parameters could be predicted to ensure continuous stability over spindle's life span. It is to be noted that that the inner working parts of the spindle studied are proprietary and its full details could not be disclosed.

\section{Equations of motion for a rotating shaft}

Consider a uniform spinning beam, exhibiting bending-bending-torsion vibrations in Fig. 1. As presented by Banerjee and $\mathrm{Su}$ [10], the in- and out-of-plane bending displacements, $u$ and $v$, along the $x$ - and $y$-axes, respectively, are coupled by the spinning speed $\Omega$. In addition, the flexural lateral displacements, $u$ and $v$, and torsional twist, $\phi$, are also coupled by bending-torsion stiffness $K$, if the beam is made of composite material. In what follows, the triply coupled differential equations governing the bending-bending-torsion vibrations are first briefly discussed, as the DSM formulation is readily available [10]. However, the coupled bending-torsion stiffness, $K$, will then be set to zero in the final equations, as in

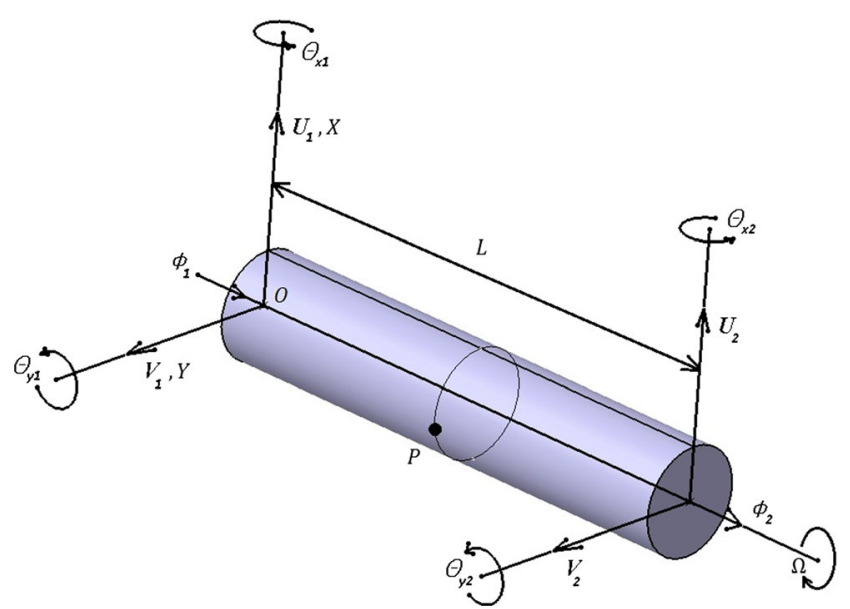

Fig. 1 Spinning beam and degrees of freedom the present study, the spinning shaft is assumed to be made of metallic (i.e. homogeneous) material.

The kinetic and potential energies, $T$ and $U$, of the spinning beam are given by [10]:

$$
\begin{aligned}
T= & \frac{1}{2} \int_{0}^{L}|v|^{2} m \mathrm{~d} z=\frac{1}{2} m \int_{0}^{L}\left[\dot{u}^{2}+\dot{v}^{2}+2 \Omega(u \dot{v}-\dot{u} v)+\Omega^{2}\left(u^{2}+v^{2}\right)\right] \\
\mathrm{d} z+\frac{1}{2} I_{\alpha} \int_{0}^{L}\left[\dot{\phi}^{2}+\Omega^{2} \phi^{2}\right] \mathrm{d} z & (\mathrm{a}-1) \\
U & =\frac{1}{2} E I \int_{0}^{L} v^{\prime \prime 2} \mathrm{~d} z+\frac{1}{2} E I \int_{0}^{L} u^{\prime \prime 2} \mathrm{~d} z+\frac{1}{2} K \int_{0}^{L} v^{\prime \prime} \phi^{\prime} \mathrm{d} z \quad(1-\mathrm{b}) \\
& +K \int_{0}^{L} u^{\prime \prime} \phi^{\prime} \mathrm{d} z+\frac{1}{2} G J \int_{0}^{L} \phi^{\prime 2} \mathrm{~d} z
\end{aligned}
$$

Using the Hamilton Principle in the usual notation state (where $t_{1}$ and $t_{2}$ are the time intervals in the dynamic trajectory and $\delta$ is the variational operator)

$\delta \int_{t_{1}}^{t_{2}}(T-U) \mathrm{d} t=0$

substituting the kinetic and potential energies of the beam in the Hamilton principle, collecting like terms and integrating by parts, the following set of equations are obtained:

$E I u^{\prime \prime} "-m \Omega^{2} u+m \ddot{u}-2 m \Omega \dot{v}+K \phi^{\prime \prime \prime}=0$,

$-2 m \Omega \dot{u}-E I v^{\prime \prime \prime}+m \ddot{v}-m \Omega^{2} v+K \phi^{\prime \prime \prime}=0$,

$\frac{1}{2} K u^{\prime \prime \prime}+\frac{1}{2} K v^{\prime \prime \prime}+\mathrm{GJ} \phi^{\prime \prime}-I_{\alpha} \ddot{\phi}+I_{\alpha} \Omega^{2} \phi=0$.

The resultant internal loads are then found to be in the form of

$S_{x}=E I u^{\prime \prime \prime}+K \phi^{\prime \prime}$, and $S_{y}=E I v^{\prime \prime \prime}+K \phi^{\prime \prime}$

for shear forces, $S_{x}$ and $S_{y}$, along ' $x$ ' and ' $y$ ' directions, respectively, and bending moments, $M_{x}$ and $M_{y}$, about ' $x$ ' and ' $y$ ' axes, respectively, are:

$M_{x}=E I v^{\prime \prime}+K \phi^{\prime}$, and $M_{y}=E I u^{\prime \prime}+K \phi^{\prime}$ 
and the torsional Torque about $z$ axis, $T$, is written as:

$T=-\left(K u^{\prime \prime}+K v^{\prime \prime}+G J \phi^{\prime}\right)$.

Assuming the harmonic motion, displacements are written as:

$u(z, t)=U(z) e^{i \omega t}, \quad v(z, t)=V(z) e^{i \omega t}, \quad \phi(z, t)=\Phi(z) e^{i \omega t}$,

where $\omega$ is frequency of oscillation, and $U(z), V(z)$ and $\Phi(z)$ are the amplitudes of $u, v$ and $\phi$, respectively. Substituting Eq. (7) into Eqs. (3-a), (3-b) and (3-c) leads to:

EI $U^{\prime \prime \prime} "-m\left(\Omega^{2}+\omega^{2}\right) U-2 i m \Omega \omega V+K \Phi^{\prime \prime \prime}=0, \quad(8-\mathrm{a})$

$2 i m \Omega \omega U-E I V^{\prime \prime} "-m\left(\Omega^{2}+\omega^{2}\right) V+K \Phi^{\prime \prime \prime}=0, \quad(8-\mathrm{b})$

$\frac{1}{2} K U^{\prime \prime \prime}+\frac{1}{2} K V+G J \Phi^{\prime \prime}-I_{\alpha}\left(\Omega^{2}+\omega^{2}\right) \Phi=0 . \quad(8-\mathrm{c})$

Introducing $\xi=z / L$ and $D=\mathrm{d} / \mathrm{d} \xi$, which are nondimensional length and the differential operator, respectively, into Eq. (3-a), (3-b) and (3-c) results in:

$\left[D^{4}-\frac{m\left(\Omega^{2}+\omega^{2}\right) L^{4}}{E I}\right] U-\frac{2 i m \Omega \omega L^{4}}{E I} V+\frac{K L}{E I} D^{3} \Phi^{\prime \prime \prime}=0, \quad(9-\mathrm{a})$

$\frac{2 i m \Omega \omega L^{4}}{E I} U+\left[D^{4}-\frac{m\left(\Omega^{2}+\omega^{2}\right) \mathrm{L}^{4}}{E I}\right] V+\frac{K L}{E I} D^{3} \Phi^{\prime \prime \prime}=0, \quad(9-\mathrm{b})$

$\frac{K}{L G J} D^{3} U+\frac{K}{L G J} D^{3} V+\left[D^{2}+\frac{I_{\alpha}\left(\Omega^{2}+\omega^{2}\right) L^{2}}{G J}\right] \Phi=0 .(9-\mathrm{c})$

One can then combine the three Eqs. (3-a), (3-b) and (3-c) above into one 10th-order differential equation, written as:

$\left[D^{10}+\mu \eta \theta D^{8}-\lambda \eta(1+\theta) D^{6}-2 \mu \lambda \theta \eta^{2} D^{4}+\lambda^{2} \varphi \theta^{2} D^{2}+\mu \lambda^{2} \eta \bar{\eta}^{2} \theta\right] W=0$

Equation (10) written in terms of $W$, is satisfied by all $U, V$ and $\Phi$, where
Substituting Eq. (12) into (10) leads to:

$r^{10}+\mu \eta \theta r^{8}-\lambda \eta(1+\theta) r^{6}-2 \mu \lambda \theta \eta^{2} r^{4}+\lambda^{2} \theta \bar{\eta}^{2} r^{2}+\mu \lambda^{2} \eta \bar{\eta}^{2} \theta=0$

$t=\frac{\Omega}{\omega}, \quad \mu=\frac{I_{\alpha} \omega^{2} L^{2}}{G J}, \quad \eta=1+t^{2}, \quad \bar{\eta}=1-t^{2}, \quad \lambda=\frac{m \omega^{2} L^{4}}{E I}, \quad \theta=\left(1-\frac{2 K^{2}}{E I G J}\right)^{-1}$

The solution of the differential Eq. (10) is sought in the form

$W=e^{r \xi}$

By solving the above Eq. (13), one obtains 10 roots for $r$. Therefore, the solutions of $U, V$ and $\Phi$ can be written as:

$U(\xi)=\sum_{j=1}^{10} P_{j} e^{r_{j} \xi}, \quad V(\xi)=\sum_{j=1}^{10} Q_{j} e^{r_{j} \xi}, \Phi(\xi)=\sum_{j=1}^{10} R_{j} e^{r_{j} \xi}$

where $\boldsymbol{r}_{j}, \boldsymbol{j}=1,2, \ldots, 10$ are the 10 roots of Eq. (13) and $\boldsymbol{P}_{j}, \boldsymbol{Q}_{j}$ and $\boldsymbol{R}_{j}, \boldsymbol{j}=1,2, \ldots, 10$ are three separate sets of 10 (possibly complex) constants. It can also be shown that [10]

$P_{j}=f_{i} R_{i}$, and $Q_{j}=g_{i} R_{i}$

where,

$f_{j}=-\frac{K L \quad r_{j}^{2}-\lambda \eta+2 i \lambda t}{E I\left(r_{j}^{4}-\lambda \eta\right)^{2}-4 \lambda^{2} t^{2}} r_{j}^{3}$, and $g_{j}=-\frac{K L \quad r_{j}^{2}-\lambda \eta-2 i \lambda t}{E I\left(r_{j}^{4}-\lambda \eta\right)^{2}-4 \lambda^{2} t^{2}} r_{j}^{3}$.

From the above solutions of $U$ and $V$, as stated in Eq. (14), the corresponding bending rotations (i.e. slopes) about $X$ - and $Y$-axes, $\Theta_{x}$ and $\Theta_{y}$, respectively, are given by

$\Theta_{x}=\frac{\mathrm{d} V}{\mathrm{~d} z}=-\frac{1}{\mathrm{~L}} \frac{\mathrm{d} V}{\mathrm{~d} \xi}=-\frac{1}{\mathrm{~L}} \sum_{10}^{j=1} r_{j} Q_{j} e^{r_{j} \xi}$, and $\quad \Theta_{y}=\frac{\mathrm{d} U}{\mathrm{~d} z}=\frac{1}{\mathrm{~L}} \frac{\mathrm{d} U}{\mathrm{~d} \xi}=\frac{1}{L} \sum_{10}^{j=1} r_{j} P_{j} e^{r_{j} \xi}$

Similar substitutions in the load Eqs. (5) and (6), leads to:

$S_{x}=\left(\frac{E I}{L^{3}} \sum_{j=1}^{10} r_{j}^{3} f_{j}+\frac{K}{2 L^{2}} \sum_{j=1}^{10} r_{j}^{2}\right) R_{j} e^{r_{j} \xi}$,

$(18-a, b)$

$S_{y}=\left(\frac{E I}{L^{3}} \sum_{j=1}^{10} r_{j}^{3} g_{j}+\frac{K}{2 L^{2}} \sum_{j=1}^{10} r_{j}^{2}\right) R_{j} e^{r_{j} \xi}$ 


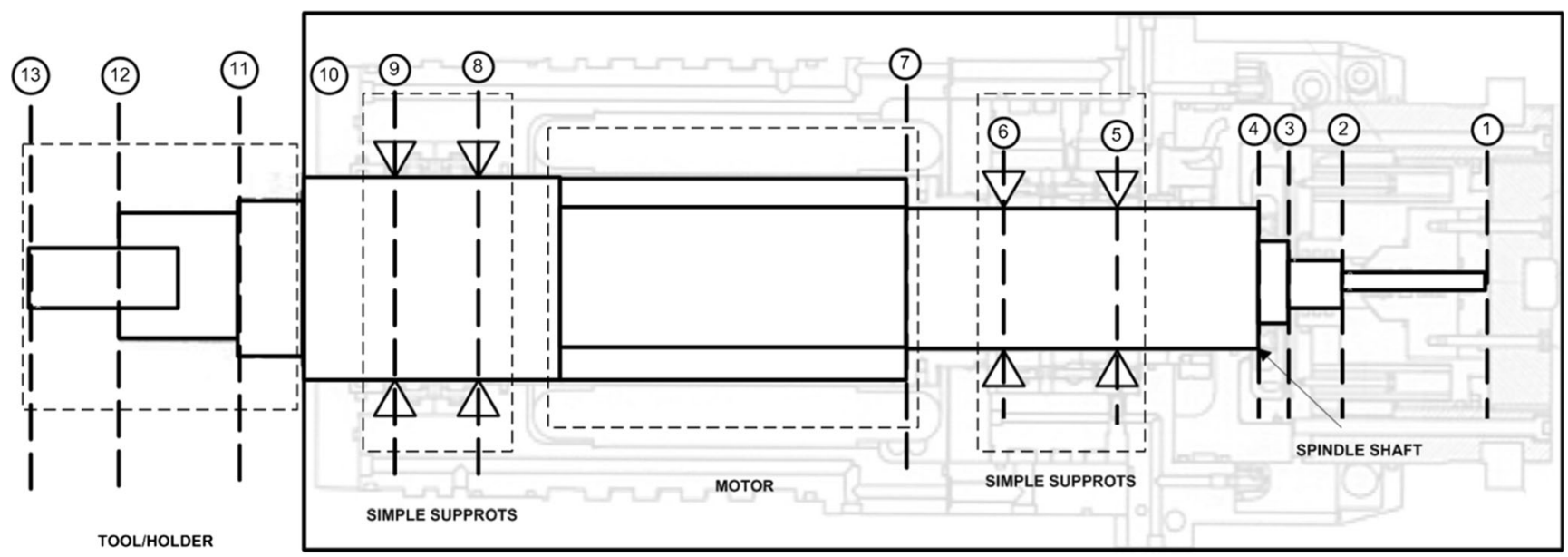

Fig. 2 Simplified spindle sections

SPINDLE CASING

$$
\begin{aligned}
M_{x} & =\left(\frac{E I}{L^{2}} \sum_{j=1}^{10} r_{j}^{2} g_{j}+\frac{K}{2 L^{2}} \sum_{j=1}^{10} r_{j}\right) R_{j} e^{r_{j} \xi}, \\
M_{y} & =\left(\frac{E I}{L^{2}} \sum_{j=1}^{10} r_{j}^{2} f_{j}+\frac{K}{2 L^{2}} \sum_{j=1}^{10} r_{j}\right) R_{j} e^{r_{j} \xi}
\end{aligned}
$$$$
T=-\left[\frac{G J}{L} \sum_{j=1}^{10} r_{j}+\frac{K}{2 L^{2}} \sum_{j=1}^{10} r_{j}^{2}\left(f_{j}+g_{j}\right)\right] R_{j} e^{r_{j} \xi} \quad(18-\mathrm{e})
$$

By introducing the boundary conditions into the governing equations (at $\xi=0$, and $\xi=1$ ), one finds

$\delta=B R$ where

$$
\begin{aligned}
& \delta=\left[\begin{array}{llllllllll}
U_{1} & V_{1} & \Theta_{x 1} & \Theta_{y 1} & \Phi_{1} & U_{2} & V_{2} & \Theta_{x 2} & \Theta_{y 2} & \Phi_{2}
\end{array}\right]^{T} \\
& R=\left[\begin{array}{lllllllllll}
R_{1} & R_{2} & R_{3} & R_{4} & R_{5} & R_{6} & R_{7} & R_{8} & R_{9} & R_{10}
\end{array}\right]^{T}
\end{aligned}
$$

\begin{tabular}{|c|c|c|c|c|c|c|c|}
\hline \multirow[b]{2}{*}{ Mode } & \multirow{2}{*}{$\begin{array}{l}\text { DSM } \\
13 \text { sections }\end{array}$} & \multicolumn{2}{|l|}{ B33 (Hz) } & \multicolumn{2}{|l|}{ C3D20R (Hz) } & \multicolumn{2}{|l|}{ C3D10M (Hz } \\
\hline & & 600 element & 1200 element & 16,965 elements & 20,122 elements & 34,465 elements & 45,645 elements \\
\hline 1 & 1392.92 & 1393.00 & 1393.00 & 1308.00 & 1308.00 & 1301.001301 .00 & \\
\hline 2 & 1393.24 & 1393.00 & 1393.00 & 1308.00 & 1308.00 & 1301.00 & 1301.00 \\
\hline 3 & 4071.02 & 4071.00 & 4071.00 & 3208.00 & 3212.00 & 3335.00 & 3322.00 \\
\hline 4 & 4071.34 & 4071.00 & 4071.00 & 3210.00 & 3212.00 & 3335.00 & 3324.00 \\
\hline Torsional & 4528.12 & 5212.00 & 5212.00 & - & - & - & - \\
\hline 5 & 5668.14 & 5669.00 & 5669.00 & 4339.00 & 4311.00 & 4322.00 & 4291.00 \\
\hline 6 & 5668.46 & 5669.00 & 5669.00 & 4341.00 & 4316.00 & 4323.004292 .00 & \\
\hline
\end{tabular}

and

$$
B=\left[\begin{array}{cccccccccc}
f_{1} & f_{2} & f_{3} & f_{4} & f_{5} & f_{6} & f_{7} & f_{8} & f_{9} & f_{10} \\
g_{1} & g_{2} & g_{3} & g_{4} & g_{5} & g_{6} & g_{7} & g_{8} & g_{9} & g_{10} \\
\beta_{1} & \beta_{2} & \beta_{3} & \beta_{4} & \beta_{5} & \beta_{6} & \beta_{7} & \beta_{8} & \beta_{9} & \beta_{10} \\
\alpha_{1} & \alpha_{2} & \alpha_{3} & \alpha_{4} & \alpha_{5} & \alpha_{6} & \alpha_{7} & \alpha_{8} & \alpha_{9} & \alpha_{10} \\
1 & 1 & 1 & 1 & 1 & 1 & 1 & 1 & 1 & 1 \\
f_{1} e^{r_{1}} & f_{2} e^{r_{2}} & f_{3} e^{r_{3}} & f_{4} e^{r_{4}} & f_{5} e^{r_{5}} & f_{6} e^{r_{6}} & f_{7} e^{r_{7}} & f_{8} e^{r_{8}} & f_{9} e^{r_{9}} & f_{10} e^{r_{10}} \\
g_{1} e^{r_{1}} & g_{2} e^{r_{2}} & g_{3} e^{r_{3}} & g_{4} e^{r_{4}} & g_{5} e^{r_{5}} & g_{6} e^{r_{6}} & g_{7} e^{r_{7}} & g_{8} e^{r_{8}} & g_{9} e^{r_{9}} & g_{10} e^{r_{10}} \\
\beta_{1} e^{r_{1}} & \beta_{2} e^{r_{2}} & \beta_{3} e^{r_{3}} & \beta_{4} e^{r_{4}} & \beta_{5} e^{r_{5}} & \beta_{6} e^{r_{6}} & \beta_{7} e^{r_{7}} & \beta_{8} e^{r_{8}} & \beta_{9} e^{r_{9}} & \beta_{10} e^{r_{10}} \\
\alpha_{1} e^{r_{1}} & \alpha_{2} e^{r_{2}} & \alpha_{2} e^{r_{2}} & \alpha_{3} e^{r_{3}} & \alpha_{4} e^{r_{4}} & \alpha_{5} e^{r_{5}} & \alpha_{e}^{r_{6}} & \alpha_{7} e_{r_{7}} & \alpha_{8} e^{r_{8}} & \alpha_{10} e^{r_{10}} \\
e^{r_{1}} & e^{r_{2}} & e^{r_{3}} & e^{r_{4}} & e^{r_{5}} & e^{r_{6}} & e^{r_{7}} & e^{r_{8}} & e^{r_{9}} & e^{r_{10}}
\end{array}\right]
$$

Table 1 Spindle natural frequencies; DSM vs. FEA for a non-spinning simply supported system 


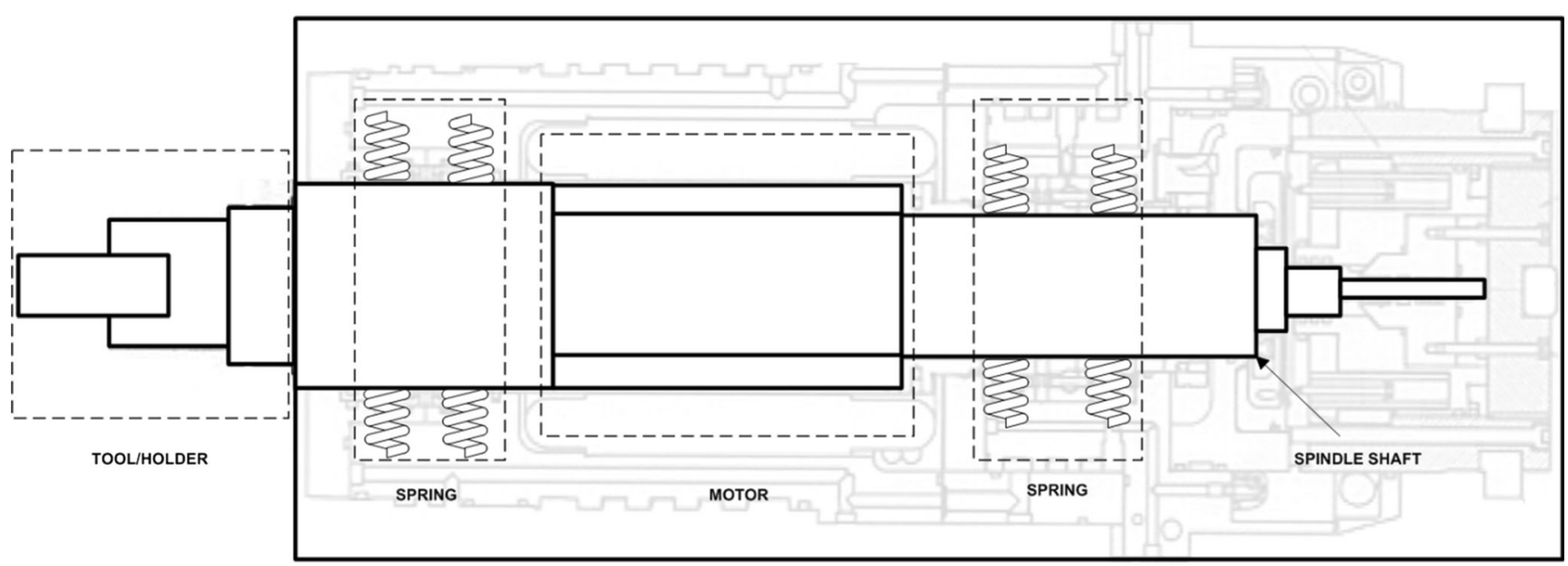

SPINDLE CASING

Fig. 3 Modified boundary conditions

and $\alpha_{i}=\frac{r_{i} f_{i}}{L}$, and $\beta_{i}=-\frac{r_{i} g_{i}}{L}$. Similar substitutions for the force equations leads to:

$F=A R$

where

$F=\left[\begin{array}{llllllllll}S_{x 1} & S_{y 1} & M_{x 1} & M_{y 1} & T_{1} & S_{x 2} & S_{y 2} & M_{x 2} & M_{y 2} & T_{2}\end{array}\right]^{T}$

$A=\left[\begin{array}{llllllllll}a_{1,1} & a_{1,2} & a_{1,3} & a_{1,4} & a_{1,5} & a_{1,6} & a_{1,7} & a_{1,8} & a_{1,9} & a_{1,10} \\ a_{2,1} & a_{2,2} & a_{2,3} & a_{2,4} & a_{2,5} & a_{2,6} & a_{2,7} & a_{2,8} & a_{2,9} & a_{2,10} \\ a_{3,1} & a_{3,2} & a_{3,3} & a_{3,4} & a_{3,5} & a_{3,6} & a_{3,7} & a_{3,8} & a_{3,9} & a_{3,10} \\ a_{4,1} & a_{4,2} & a_{4,3} & a_{4,4} & a_{4,5} & a_{4,6} & a_{4,7} & a_{4,8} & a_{4,9} & a_{4,10} \\ a_{5,1} & a_{5,2} & a_{5,3} & a_{5,4} & a_{5,5} & a_{5,6} & a_{5,7} & a_{5,8} & a_{5,9} & a_{5,10} \\ a_{6,1} & a_{6,2} & a_{6,3} & a_{6,4} & a_{6,5} & a_{6,6} & a_{6,7} & a_{6,8} & a_{6,9} & a_{6,10} \\ a_{7,1} & a_{7,2} & a_{7,3} & a_{7,4} & a_{7,5} & a_{7,6} & a_{7,7} & a_{7,8} & a_{7,9} & a_{7,10} \\ a_{8,1} & a_{8,2} & a_{8,3} & a_{8,4} & a_{8,5} & a_{8,6} & a_{8,7} & a_{8,8} & a_{8,9} & a_{8,10} \\ a_{9,1} & a_{9,2} & a_{9,3} & a_{9,4} & a_{9,5} & a_{9,6} & a_{9,7} & a_{9,8} & a_{9,9} & a_{9,10} \\ a_{10,1} & a_{10,2} & a_{10,3} & a_{10,4} & a_{10,5} & a_{10,6} & a_{10,7} & a_{10,8} & a_{10,9} & a_{10,10}\end{array}\right]$

and

$a_{1, j}=\left(\frac{E I}{L^{3}} r_{j} f_{j}+\frac{K}{L^{2}}\right) r_{j}^{2}, \quad a_{2, j}=\left(\frac{E I}{L^{3}} r_{j} g_{j}+\frac{K}{L^{2}}\right) r_{j}^{2} \quad(26-\mathrm{a}, \mathrm{b})$

$a_{3, j}=\left(\frac{E I}{L^{2}} r_{j} g_{j}+\frac{K}{L}\right) r_{j}, \quad a_{4, j}=-\left(\frac{E I}{L^{2}} r_{j} f_{j}+\frac{K}{L}\right) r_{j} \quad(26-\mathrm{c}, \mathrm{d})$

$a_{5, j}=-\left[\frac{G J}{L}+\frac{K}{L^{2}} r_{j}\left(f_{j}+g_{j}\right)\right] r_{j}, \quad a_{6, j}=-\left(\frac{E I}{L^{3}} r_{j} f_{j}+\frac{K}{L^{2}}\right) r_{j}^{2} e^{r_{j}}$

$(26-\mathrm{e}, \mathrm{f})$ $a_{7, j}=-\left(\frac{E I}{L^{3}} r_{j} g_{j}+\frac{K}{L^{2}}\right) r_{j}^{2} e^{r_{j}}, \quad a_{8, j}=-\left(\frac{E I}{L^{2}} r_{j} g_{j}+\frac{K}{L}\right) r_{j} e^{r_{j}}(26-\mathrm{g}, \mathrm{h})$

$a_{9, j}=\left(\frac{E I}{L^{2}} r_{j} f_{j}+\frac{K}{L}\right) r_{j} e^{r_{j}}, \quad a_{10, j}=\left[\frac{G J}{L}+\frac{K}{L^{2}} r_{j}\left(f_{j}+g_{j}\right)\right] r_{j} e^{r_{j}}$

$(26-\mathrm{i}, \mathrm{j})$

The DSM of a spinning beam element, $K^{\mathrm{e}}(\omega)$, is then derived by eliminating $R$ from Eqs. (19) and (23), leading to:

$F=K^{\mathrm{e}}(\omega) \delta$

where $K^{\mathrm{e}}(\omega)$, is the frequency-dependent DSM for the triply coupled vibrations of a uniform spinning beam, written as:

$K^{\mathrm{e}}(\omega)=A B^{-1}$

The element DSMs are then assembled to find the global dynamic stiffness matrix, $K(\omega)$. Enforcing the system boundary conditions and setting force vector $\mathbf{F}=0$ (for free vibrations), then leads to the following nonlinear eigenproblem:

$K(\omega) \delta=0$

The solution of the problem then consist of searching for system natural frequencies, $\omega$, satisfying:

$|K(\omega)|=0$ 


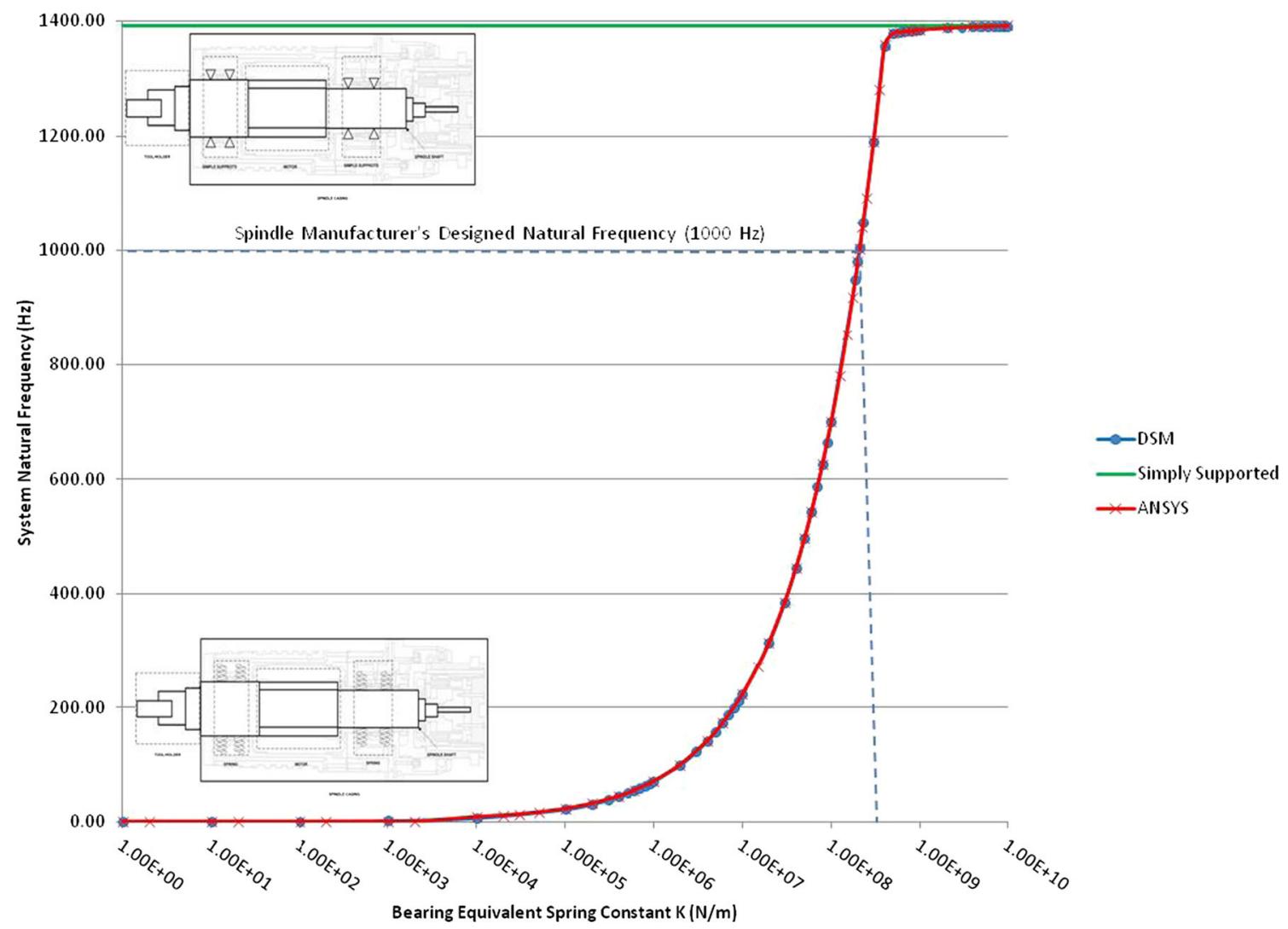

Fig. 4 System natural frequency vs. bearing equivalent spring constant, $K_{\mathrm{s}}$, for a high speed spindle (in log scale)

The corresponding modes of free vibrations, $\delta(20)$, are then extracted from expression (29).

\section{Numerical results}

The DSM formulation and the resulting element matrices (28) were implemented in a MATLAB ${ }^{\circledR}$-based code. The DSM formulation was first validated for the free flexural and torsional vibrations of simple cases of non-spinning uniform and homogeneous beams, for which exact frequency results are available in the open literature [12]. As expected, the DSM fundamental frequencies, obtained using a single DSM element were found to be in excellent agreement with exact data. In addition, the DSM frequency results were also validated against FEM-based ABAQUS ${ }^{\circledR}$ commercial software [13] and an in-house FEM code [14].

Once the correctness of the DSM method is established, a real spindle system (Fig. 2) was modelled, where the bearings were modelled as simply supported frictionless pins. As it was mentioned previously, the inner working parts of the spindle are proprietary. Therefore, a low-resolution background picture has been intentionally used solely for the presentation purposes (Fig. 2). As the spindle system in hand is made of metallic, homogeneous material, the bending-torsion coupling stiffness, $K$, must be set to a small value close to zero, making torsional and bending vibrations of the system uncoupled; the properties of tooling steel were used for all

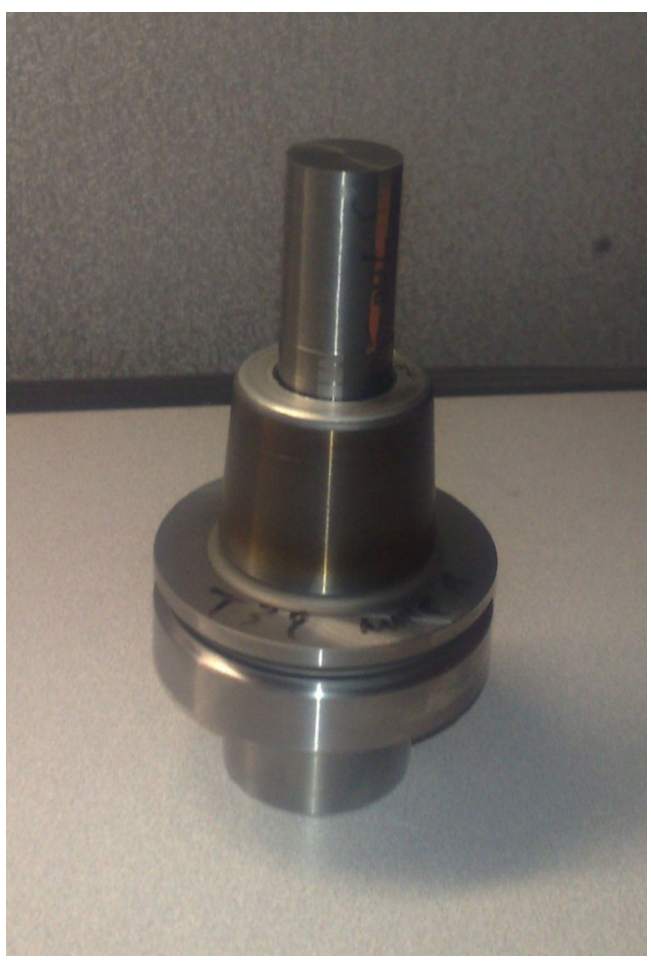

Fig. 5 Blank tool 
Fig. 6 a Blank tool in spindle and $\mathbf{b}$ experimental equipment
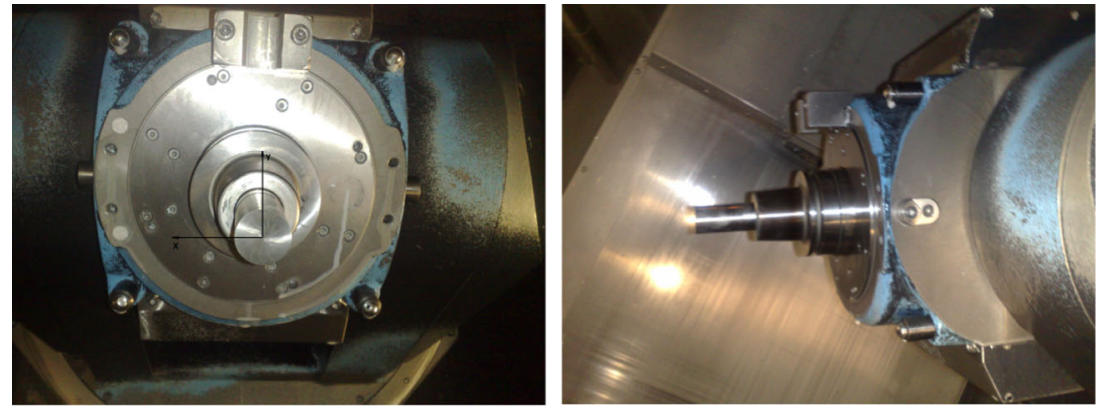

a) Blank Tool in Spindle

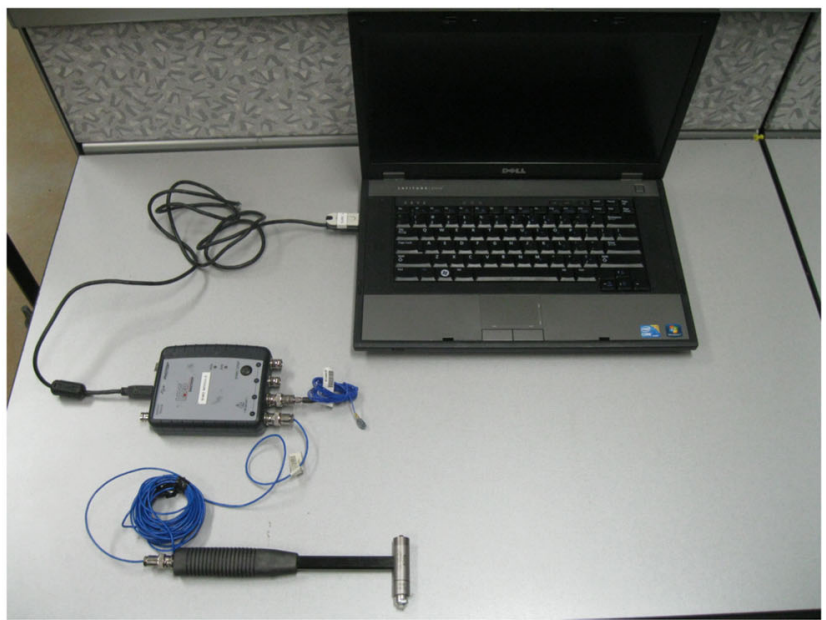

b) Experimental Equipment

Production Spindle Natural Frequency vs. Hours

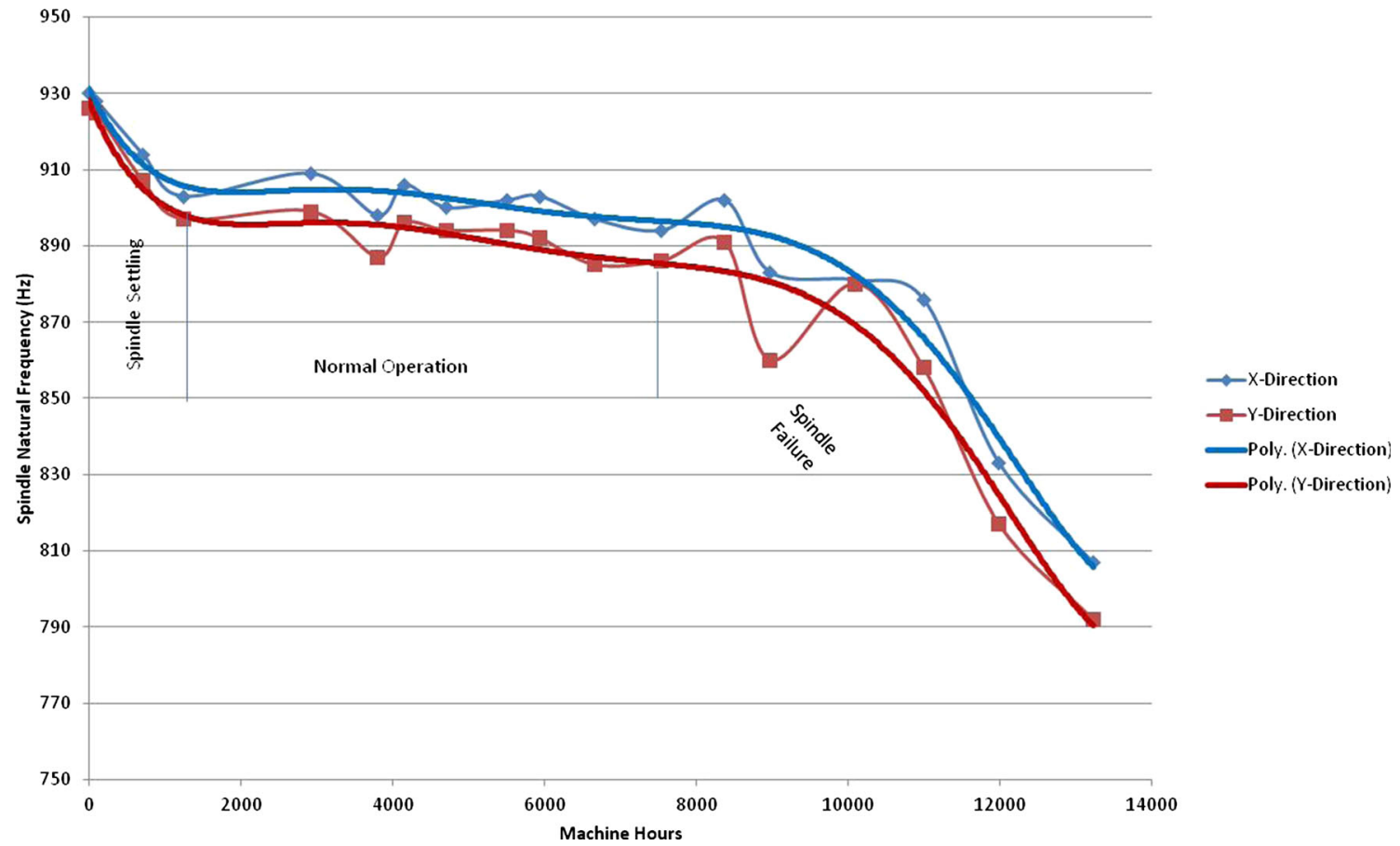

Fig. 7 Production spindle natural frequency vs. machine hours 
Prove-out Spindle 1 Natural Frequency vs. Hours

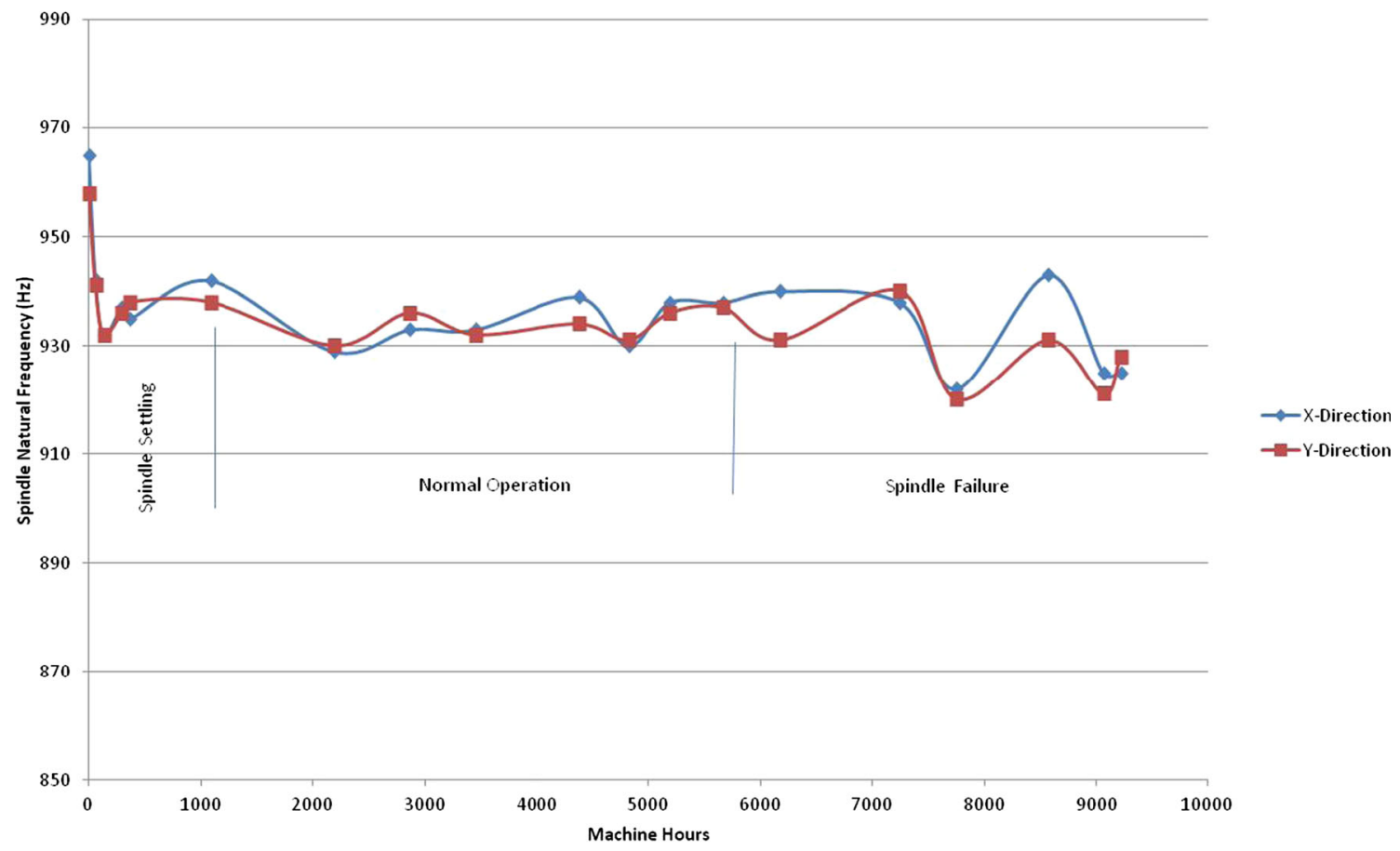

Fig. 8 Prove-out spindle 1 fundamental frequencies vs. machine hours

sections. Furthermore, to analyse the non-spinning configuration at hand, spinning speed must vanish. However, it is worth noting that due to the nature of the DSM formulation [10], $\Omega$ cannot be set directly to zero. Therefore, the spinning speed is set to a very small value, approaching zero $(\Omega \approx 0)$.
The DSM frequency results were compared with those obtained from the models generated using a conventional FEMbased commercial software (ABAQUS ${ }^{\circledR}$ ) reported by Sada [13] (see Table 1). Sada used several different FEM element types and mesh sizes to ensure the model convergence; beam

Prove-out Spindle 2 Natural Frequency vs. Hours

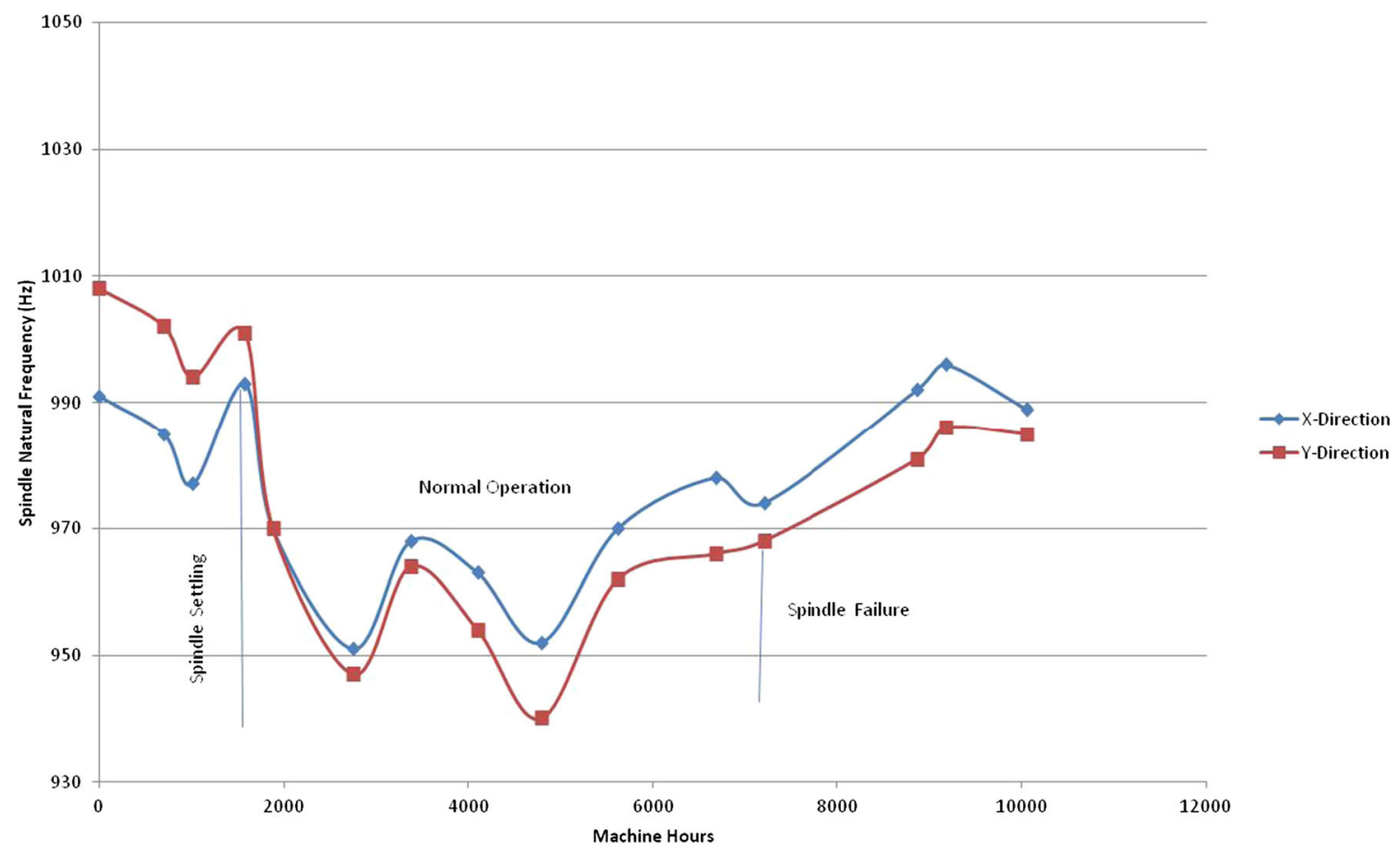

Fig. 9 Prove-out spindle 2 fundamental frequencies vs. machine hours 
Prove-out Spindle 3 Natural Frequency vs. Hours

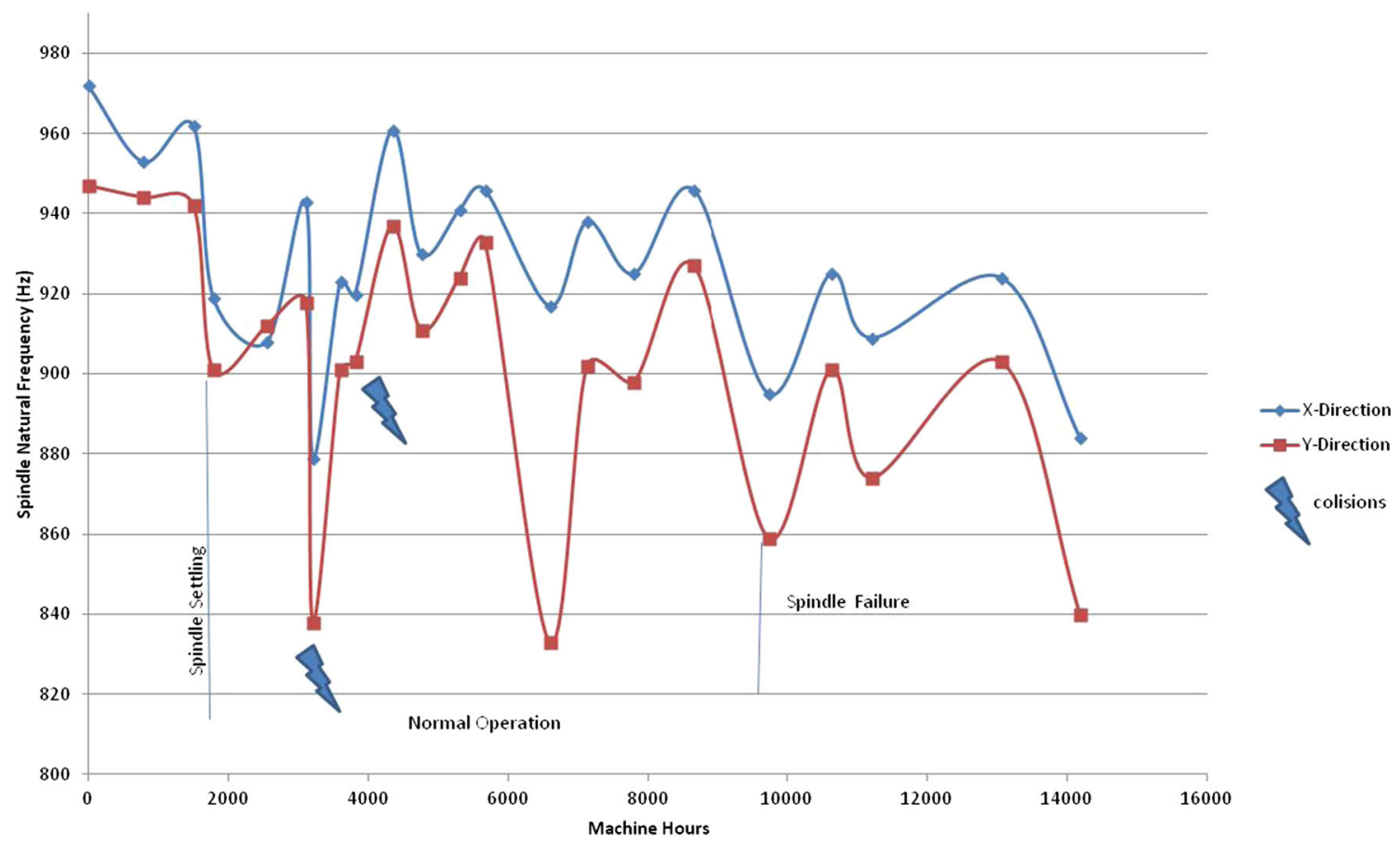

Fig. 10 Prove-out spindle 3 natural frequency vs. machine hours

element B33 uses a two-node cubic beam, C3D20R is a general-purpose quadratic brick element, and C3D10M is a general-purpose tetrahedral element. The first six bending natural frequencies and fundamental torsional frequency of the system were evaluated (see Table 1 for results). Unlike the conventional FEM formulation, the DSM method results in exact solution for all natural frequencies of spinning (and nonspinning) uniform beams. Therefore, as the spindle system at hand is made of 12 uniform sections, only a 12 -element piecewise-uniform (stepped) DSM model was required. As also discussed earlier in this paper, this is also one of the main advantages of the DSM over the conventional FEM methods [14-17]. The frequency results obtained from conventional FEM beam element (B33 in ABAQUS ${ }^{\circledR}$ ) yielded the best agreement with the DSM results, which can be explained by the fact that both elements share the same beam theories, where the three-dimensional (3D) and shear effects are neglected. However, the flexural fundamental frequency values obtained based on the simply supported bearing boundary conditions were found to be different from the experimental and nominal values reported by the manufacturer, i.e. $1393 \mathrm{~Hz}$ compared to nominal value of $1000 \pm 50 \mathrm{~Hz}$.

\subsection{Calibrated dynamic stiffness matrix model}

As mentioned earlier, all the DSM and various FEM spindle models [13], with bearing boundary conditions modelled as simply supported pins, lead to larger fundamental flexural frequency than the nominal value, i.e. rigid bearings.
However, the bearings are not fully rigid and have some inherent flexibility (see, e.g. [3] for a review on bearings stiffness calculation). Therefore, the proposed DSM model was then modified to account for the bearing flexibility, where based on the small displacement theory, the rigid, pin and simple supports were replaced by linear spring elements (Fig. 3). The spring stiffness values, $K_{\mathrm{s}}$, assumed to be identical for all bearings were then varied (Fig. 4), and a CDSM method was developed to achieve a fundamental frequency equivalent to the spindle system's fundamental frequency reported by the manufacturer.

As can be observed from Fig. 4, as the spring stiffness value increases, the natural frequency of the system increases. The natural frequency then levels out and reaches an asymptote as the springs start behaving more like simple supports at high values of spring stiffness. Figure 4 also shows that, at spring stiffness value of $K_{\mathrm{s}}=2.1 \times 10^{8} \mathrm{~N} / \mathrm{m}$, the system achieves the natural frequency reported by the spindle manufacturer $(1000 \pm 50 \mathrm{~Hz})$. This value of spring stiffness is used for any further analysis of the system. These results were also confirmed using ABAQUS ${ }^{\circledR}$ software [13].

Using the graph in Fig. 4, and the usable range of the spindle at hand (between 790 and $1050 \mathrm{~Hz}$ ), it was found that the relationship between the spindle fundamental frequency (in Hertz) and the bearings stiffness (in $\mathrm{N} / \mathrm{m}$ ) can be best represented as follows:

$K_{s}=246 \omega_{n}^{2}-5.9319 \times 10^{4} \omega_{n}+2.1408 \times 10^{7}$ 
Stability Chart for a Varying Natural Frequency Spindle

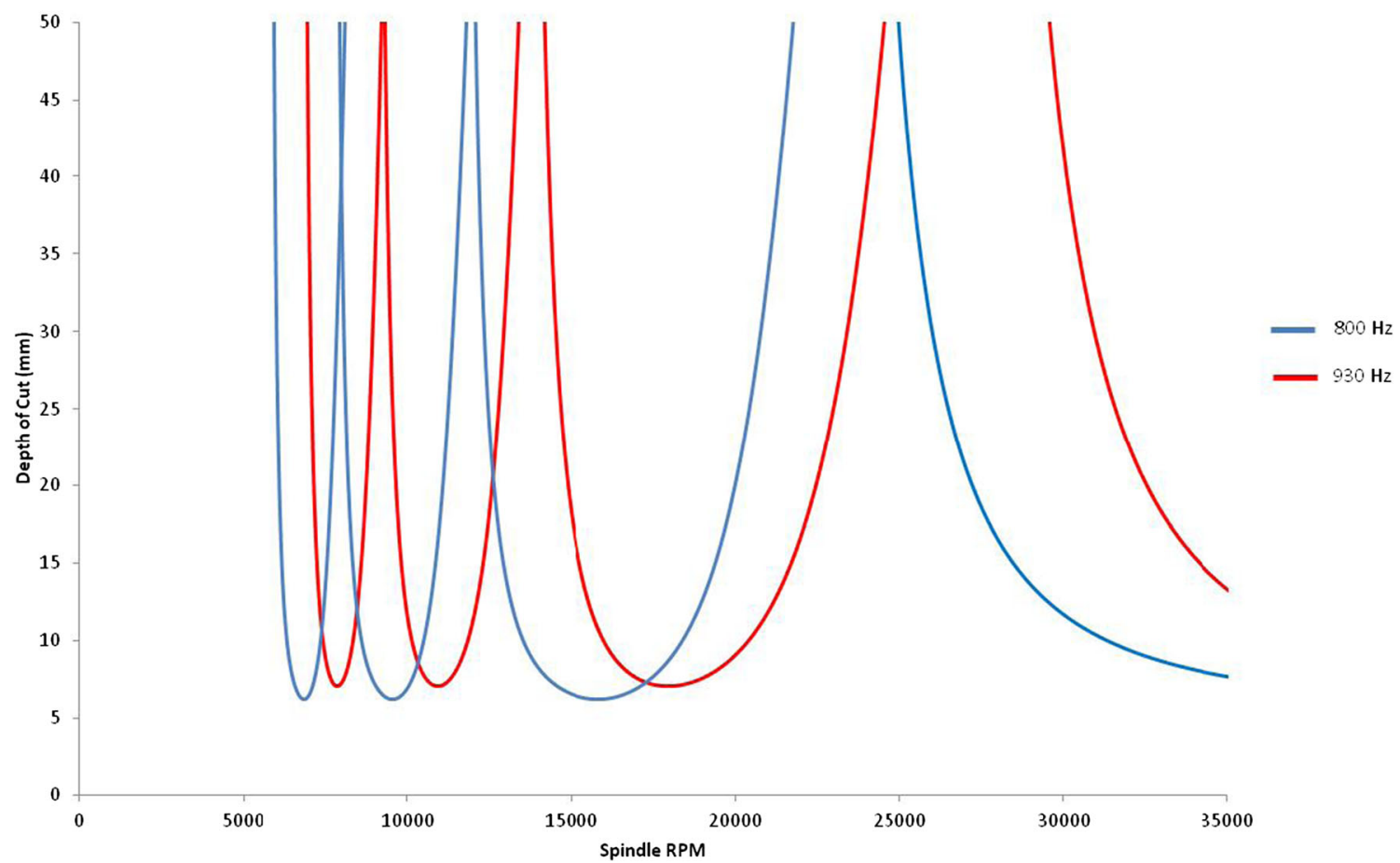

Fig. 11 Stability chart for a varying natural frequency spindle (two tooth cutter)

developed based on the assumption that all the bearings have the same stiffness coefficient, and would wear evenly and at the same time as the spindle ages. Consequently, if an equation for the spindle fundamental frequency in terms of system's age is established, i.e. $\omega_{n}=f$ (machine hours), then one could use it in conjunction with Eq. (31), to develop relationship between $K_{\mathrm{s}}$ and the spindle age. Therefore, as it will be further discussed in the next section, experimental data were collected to observe the change in the spindle fundamental frequency over time. To this end, frequency response function (FRF)-based modal analysis was carried out through tap testing, and the system natural frequencies were calculated. The resulting experimentally evaluated spindle's fundamental frequency is then used to calculate the equivalent bearing stiffness constants, $K_{\mathrm{s}}$, and spindle system overall milling stiffness, $K_{\text {theo. }}$. The resulting data can then be used to generate the stability lobes in order to identify the shift in the stable vs. unstable region caused by spindle ageing, i.e. reduced bearing stiffness.

\section{Experimental procedure}

The experimentally evaluated FRF data were collected for several machines over a period of time. A 1-in diameter blank tool with a 2-in protrusion was used. A typical shrink fit tool holder was also used. This type of holder was selected for its rigid contact surface with the tool (Fig. 5).
The tool and holder were inserted into the spindle. The spindle was set to be horizontal or neutral position shown in Fig. 6a. The accelerometer was then attached to the edge of the tool holder using the wax supplied by the kit. The spindle was struck 10 times in the $x$ direction, and the average FRF graph was generated. Similarly, the spindle was also tested along the $y$ direction.

The experimental equipment is comprised of (see Fig. 6b):

1. 352A21 (light, $0.8 \mathrm{~g}$ ) accelerometer

2. $086 \mathrm{C} 04$ ( $5000 \mathrm{~N}$ hammer) hammer

3. SIM3 module photon + data acquisition

4. Metalmax Software (TXF).

The natural frequency of the spindle was calculated verses the machine time used over the life cycle of the spindle. Metal Max Software was used, and several different spindles were monitored.

Table 2 Spindle vibrational characteristics using different tools

\begin{tabular}{lllll}
\hline Tool & Direction & Natural frequency $(\mathrm{Hz})$ & Damping & $K(\mathrm{~N} / \mathrm{m})$ \\
\hline IMCO & $x$ & 893.921 & 0.028267 & $4.3226 \mathrm{E}+07$ \\
& $y$ & 878.540 & 0.031472 & $3.9634 \mathrm{E}+07$ \\
Helical & $x$ & 889.526 & 0.030877 & $4.2798 \mathrm{E}+07$ \\
& $y$ & 879.639 & 0.030183 & $3.9895 \mathrm{E}+07$ \\
Merlin & $x$ & 860.596 & 0.039788 & $3.9222 \mathrm{E}+07$ \\
& $y$ & 859.497 & 0.036643 & $3.9236 \mathrm{E}+07$ \\
\hline
\end{tabular}




\section{Stability Chart (IMCO Tool)}

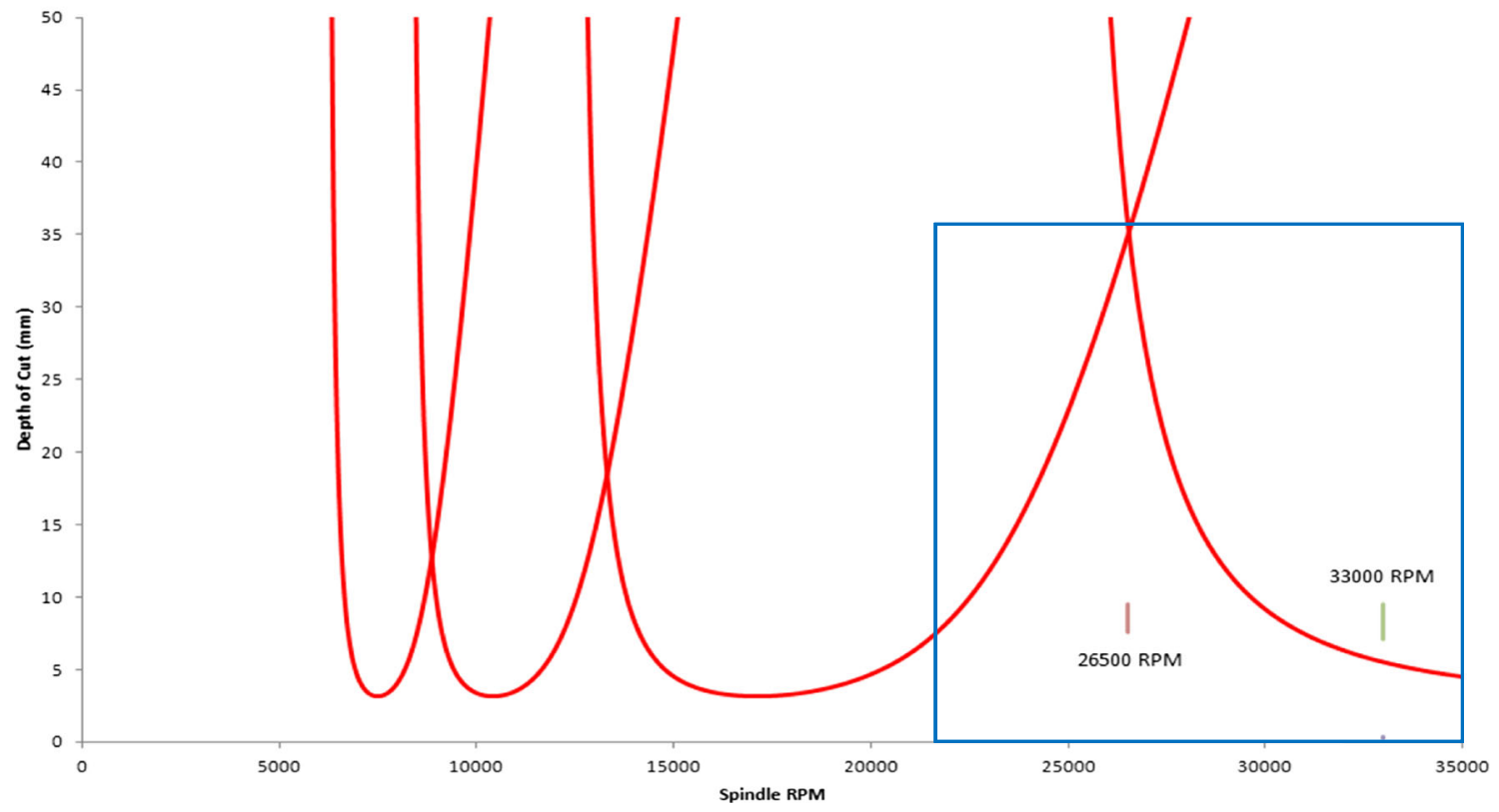

Fig. 12 Stability chart with test cuts (IMCO tool)

\section{Stability Chart (IMCO Tool)}

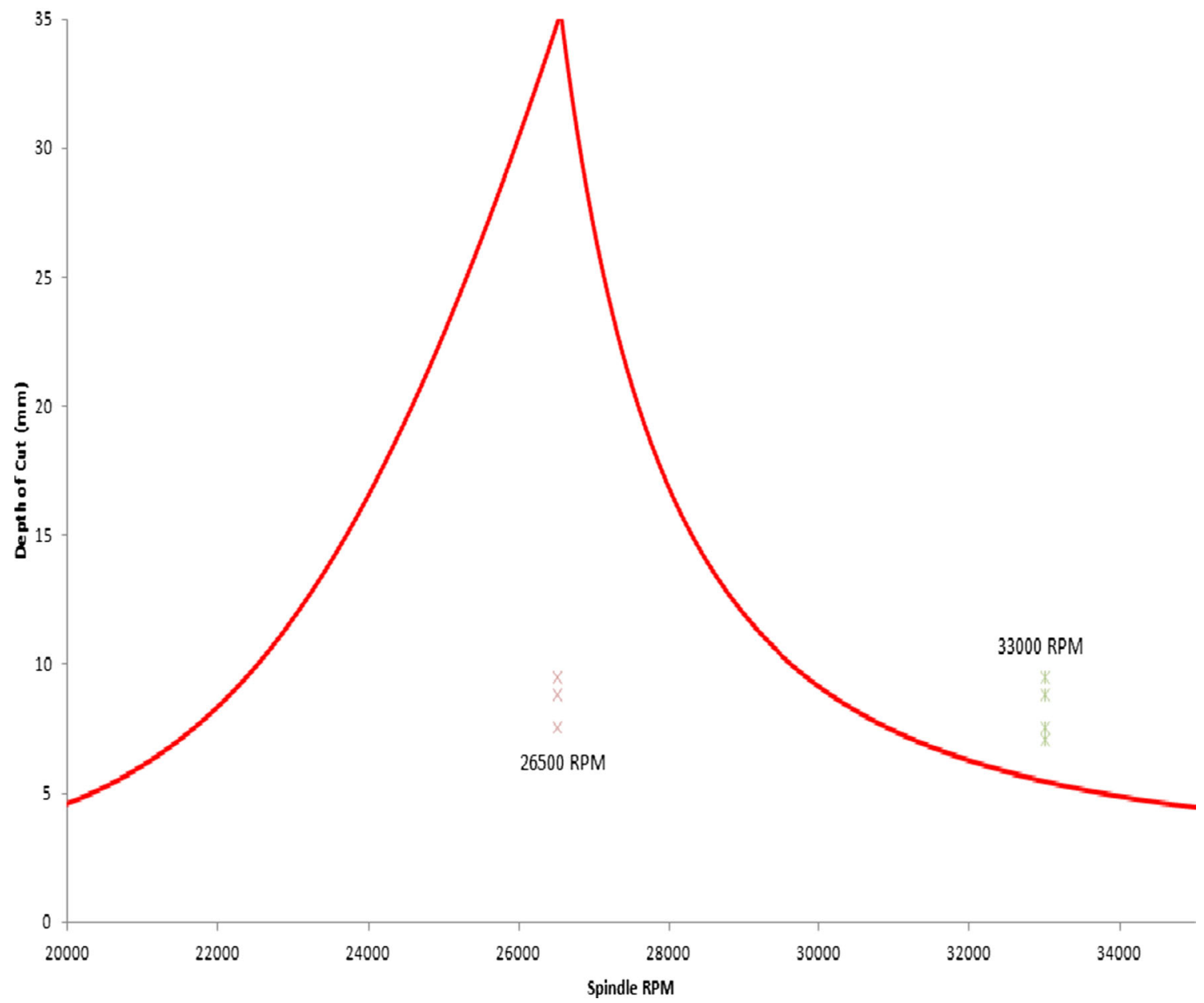

Fig. 13 Stability chart with test cuts (IMCO tool) zoomed in 


\section{Stability Chart (Helical Tool)}

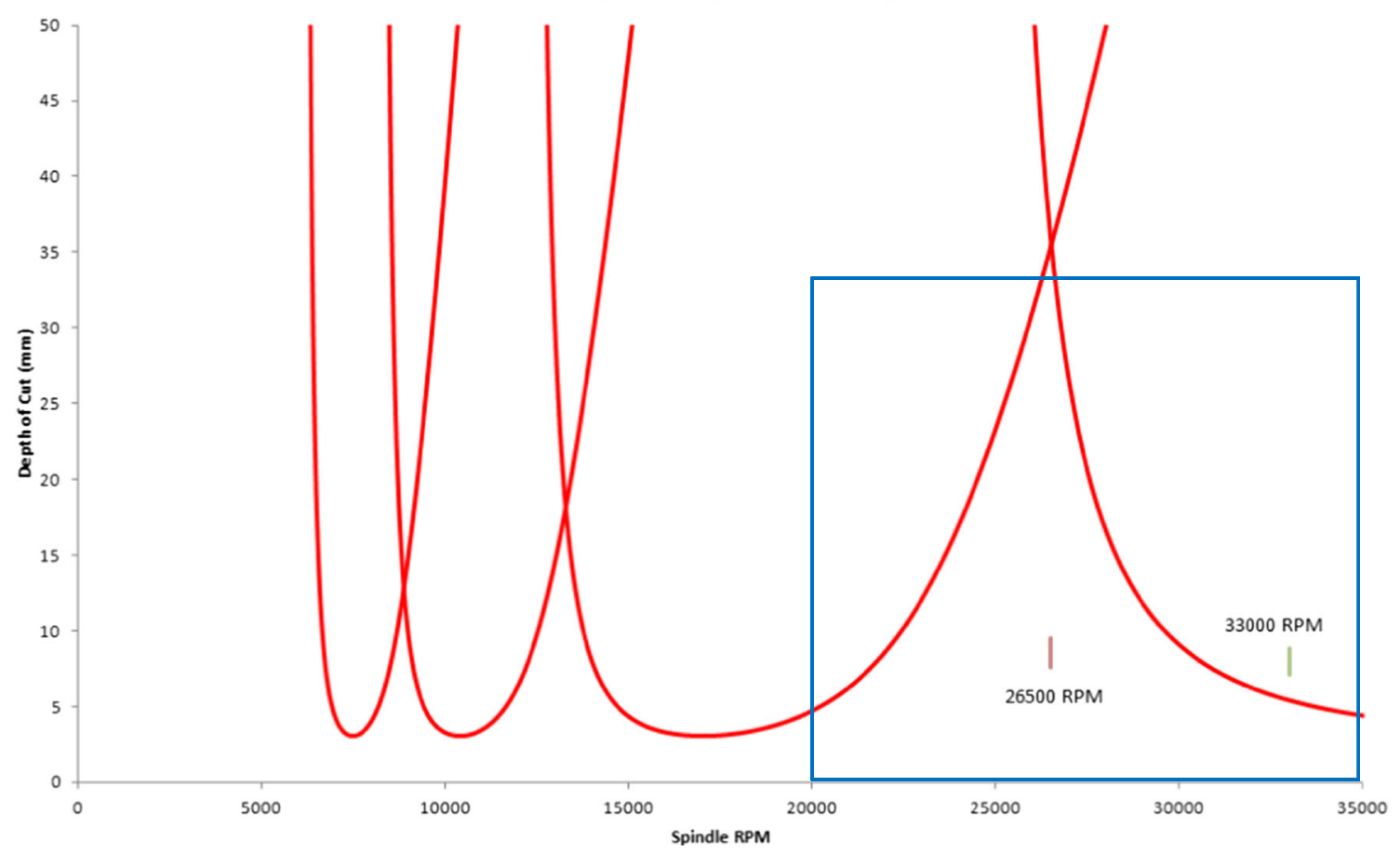

Fig. 14 Stability chart with test cuts (helical tool)

A production spindle, running similar type jobs over its life span, was the first to be investigated. The spindle has never been abused or ran over the manufacturers' recommended parameters. It has never alarmed out due to vibrations, nor had any collisions since the programme running the production machines has been thoroughly tested and fine-tuned. Therefore, the change in the spindle natural frequency can be assumed to be due to bearing wear from typical use. The natural frequency changed from $930 \mathrm{~Hz}$ to just below $800 \mathrm{~Hz}$ over the spindle's life span, i.e. 13,000 h (see Fig. 7). It is also evident that the natural frequency change is gradual and predictable. The deviation between the system's vibrational behaviour (i.e. natural frequencies) in $x$ and $y$ directions can be associated with uneven wear of the bearings caused by unequal use of spindle in different directions. The $R^{2}$ values for the fitted curves in the $x$ and $y$ directions are 0.974 and 0.957 , respectively.

The second type of spindles investigated was a prove-out spindle. A prove-out spindle is one that is used to run new computer numerical control (CNC) programmes for the first time, in order to validate the parameters specified by the programmer. These particular spindles have not gone through any collisions; however, they have alarmed out due to vibration alerts due to aggressive machining, whose parameters have been updated in subsequent runs. Two spindles of this type were monitored and, as can be seen in Figs. 8 and 9, the fundamental frequencies start at the nominal value $(1000 \pm$ $50 \mathrm{~Hz}$ ), and changes of fundamental frequency vs. machine hours (i.e. spindle age) in both cases are observed to be gradual, although not fully predictable.

The final spindle studied was a prove-out spindle similar to the second type, except that it has faced multiple collisions. This happens when the tool collides with the material or the fixture holding the material while moving around the machine. These collisions usually cause significant damage to the internal components of the spindles, particularly bearings. It is also known that these collisions change the vibrational characteristics of the spindle significantly. As can be observed from Fig. 10, in this case, the changes are erratic and unpredictable, especially around the collision point. It is suspected that, when collisions happen, the bearings deform excessively, leading to permanent distortion and change of their form, which in turn causes them to seize or loosen on the spindle shaft. Consequently, as the spindle is being used and the bearings undergo further wear, the system's vibrational response would spike or drop.

Referring to Figs. 7, 8, 9 and 10, it was also determined that the spindle life can be broken down into three major sections, namely spindle settling, normal operation and spindle failure.

The 'spindle settling' zone happens when the spindle is newly installed and has been turned on for the first time. At this stage, all the components are settling and wearing together. It is observed that, at this stage, the natural frequency changes very rapidly with time (3-5\% decrease).

Roughly after 1000-2000 h, the 'normal operation' zone starts. If the spindle was used with care and was not subjected 


\section{Stability Chart (Helical Tool)}

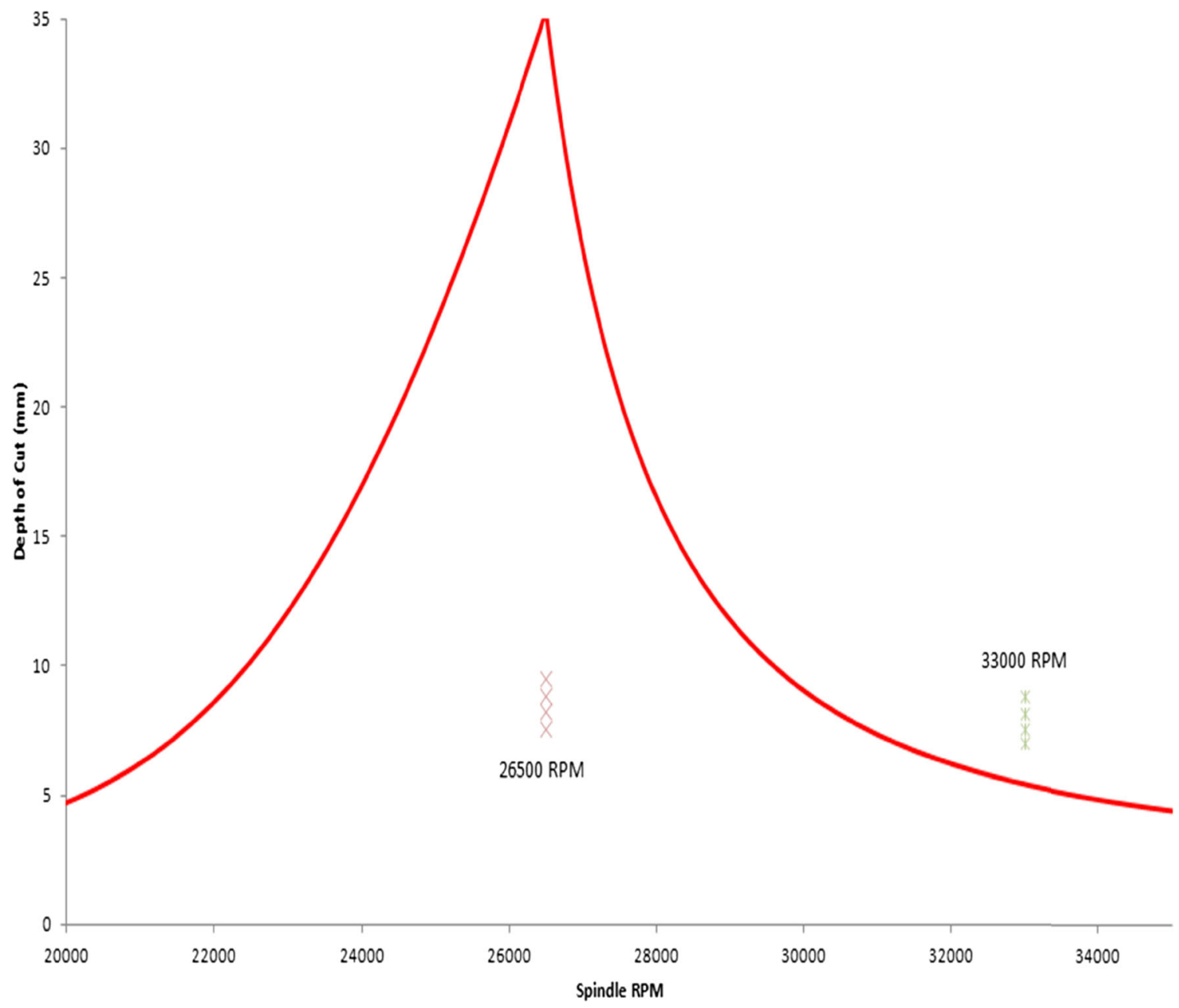

Fig. 15 Stability chart with test cuts (helical tool) zoomed in

to high vibration levels or collisions, the change in natural frequency in the 'normal operation' zone would then be gradual and predictable, i.e production spindle (Fig. 7). Otherwise, the changes can be erratic and unpredictable.

Finally, the 'spindle failure zone' is where the spindle behaviour is no longer predictable and varies inconsistently. At this stage, the spindle is approaching the end of its life cycle and is no longer dependable.

Referring to Figs. 7, 8, 9 and 10, in general, and focussing on the production spindle (Fig. 7), in particular, one notices that, once the spindle has been settled, the natural frequency stays within a very specific range that does not change significantly (max. $10 \%$ ). This behaviour is observed to last for about $5000-7000 \mathrm{~h}$. After this period, the spindle goes into failure mode, i.e. when the natural frequency is unpredictable and can spike up or drop down. At this stage, the spindle needs to be replaced. Therefore, it can be suggested that, instead of tap testing the spindle constantly (e.g. once a week or once a month, as commonly carried out during the spindle's normal operation), one can tap test the system at its brand new stage.
Then, after the settling period (drops 3-5\% drop in the natural frequency), the spindle can then be used without further interruptions, and possibly checked every $2000 \mathrm{~h}$ looking for the failure zone. Within the spindle's 'normal operation' zone, one could then account for a reduction in the natural frequency from its brand new/nominal value (e.g. $10 \%$ ) to evaluate the stability lobes. Potential sources of error in tap test data include, but not limited to, repeatability of the hammer strikes, overall system temperature and the mass of the accelerometer attached to the monument tool while testing.

In what follows, a procedure is presented that can be used in conjunction with the presented CDSM method, to evaluate the shift in the stability lobes caused by the decreased fundamental frequency of the system, over its 'normal operation' zone. As a result, it will be possible to evaluate safe machining parameters, or predict updated values, satisfying the chatterfree, stable machining for the life of the spindle until it fails, i.e. once the failure (unpredictable) zone has been reached. The application of the proposed procedure is demonstrated through the above-mentioned 'production spindle'. 


\section{Stability Chart (Merlin Tool)}

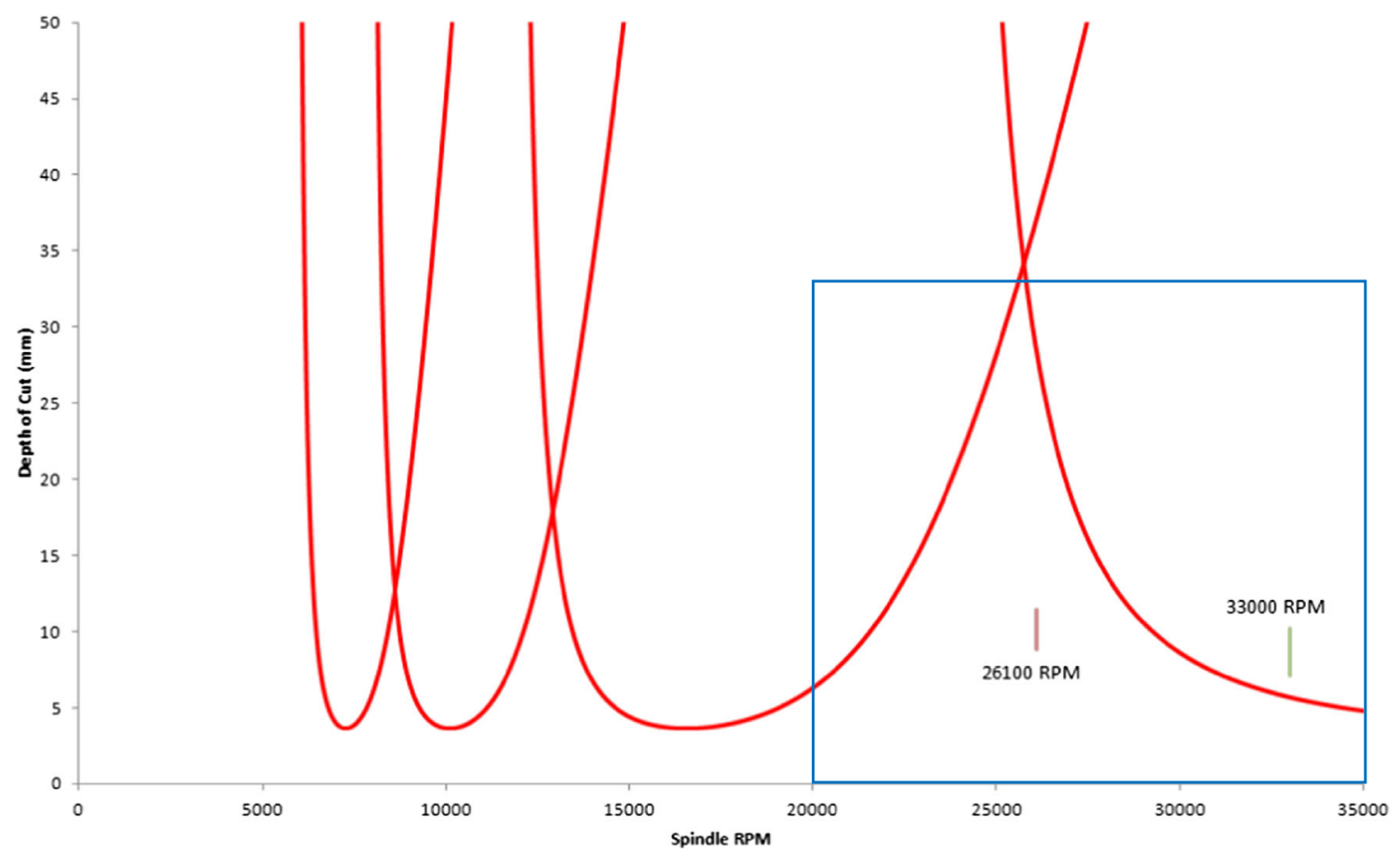

Fig. 16 Stability chart with test cuts (Merlin tool)

\section{Stability lobes}

Using the experimental data presented in the previous section and the method described in [16], the stability lobes are calculated for the production spindle, for two different frequencies (i.e. 930 and $800 \mathrm{~Hz}$ ). The $930-\mathrm{Hz}$ frequency is the frequency measured at the beginning of the life cycle of the spindle, and the $800 \mathrm{~Hz}$ represents the frequency of the spindle at the last test before the spindle was no longer operational.

Following the procedure presented in [16], the stability lobes are generated based on a spindle system modelled as a 1-DOF, subjected to end force. However, as discussed earlier in the paper, the spindle models investigated here consist of continuous DSM elements, or large number of beam/3D FEM elements, and the supporting bearings represented by multiple linear spring elements. Therefore, one needs to first convert the rotating system to a spring damper system, in order to evaluate the equivalent stiffness of the entire system, $K_{\text {theo. }}$. In what follows, the process of evaluating $K_{\text {theo }}$ is briefly presented.

The dynamic stiffness matrix of the system is first converted to a static stiffness matrix by linearising the nonlinear eigenvalue problem of the system, as described by Hashemi [17]. Based on this method, the static stiffness of the system can be evaluated at zero frequency (i.e. no vibration, $\omega=0)$, or in the neighbourhood of any given frequency [18].
Consider a very small natural frequency in the vicinity of zero $(\omega \approx 0)$, referred to as $\omega_{\mathrm{L}}$. A very small frequency increment, $\Delta \omega$, is then added to $\omega_{\mathrm{L}}$, to find

$\omega_{U}=\omega_{L}+\Delta \omega$

and an average frequency value, $\bar{\omega}$, is then defined as:

$\bar{\omega}=\left(\omega_{U}+\omega_{L}\right) / 2$

The eigenvalue problem (29) can then be linearised, in the vicinity of $\bar{\omega}$, written as:

$[K(\bar{\omega})]=[\boldsymbol{K}]-\bar{\omega}^{2}[\boldsymbol{M}]$

where $K(\bar{\omega})$ is the system DSM, and $[\boldsymbol{K}]$ and $[\boldsymbol{M}]$ are the static stiffness and mass matrices, respectively, evaluated in the vicinity of $\bar{\omega}$ frequency. The mass matrix of the system can then be written as:

$[\boldsymbol{M}]=-\frac{\mathrm{d}[K(\bar{\omega})]}{\mathrm{d}\left(\bar{\omega}^{2}\right)}$

Finally, substituting Eq. (35) back into Eq. (34), the system static stiffness matrix is obtained as:

$[\boldsymbol{K}]=[K(\bar{\omega})]+\bar{\omega}^{2}[M(\bar{\omega})]$ 


\section{Stability Chart (Merlin Tool)}

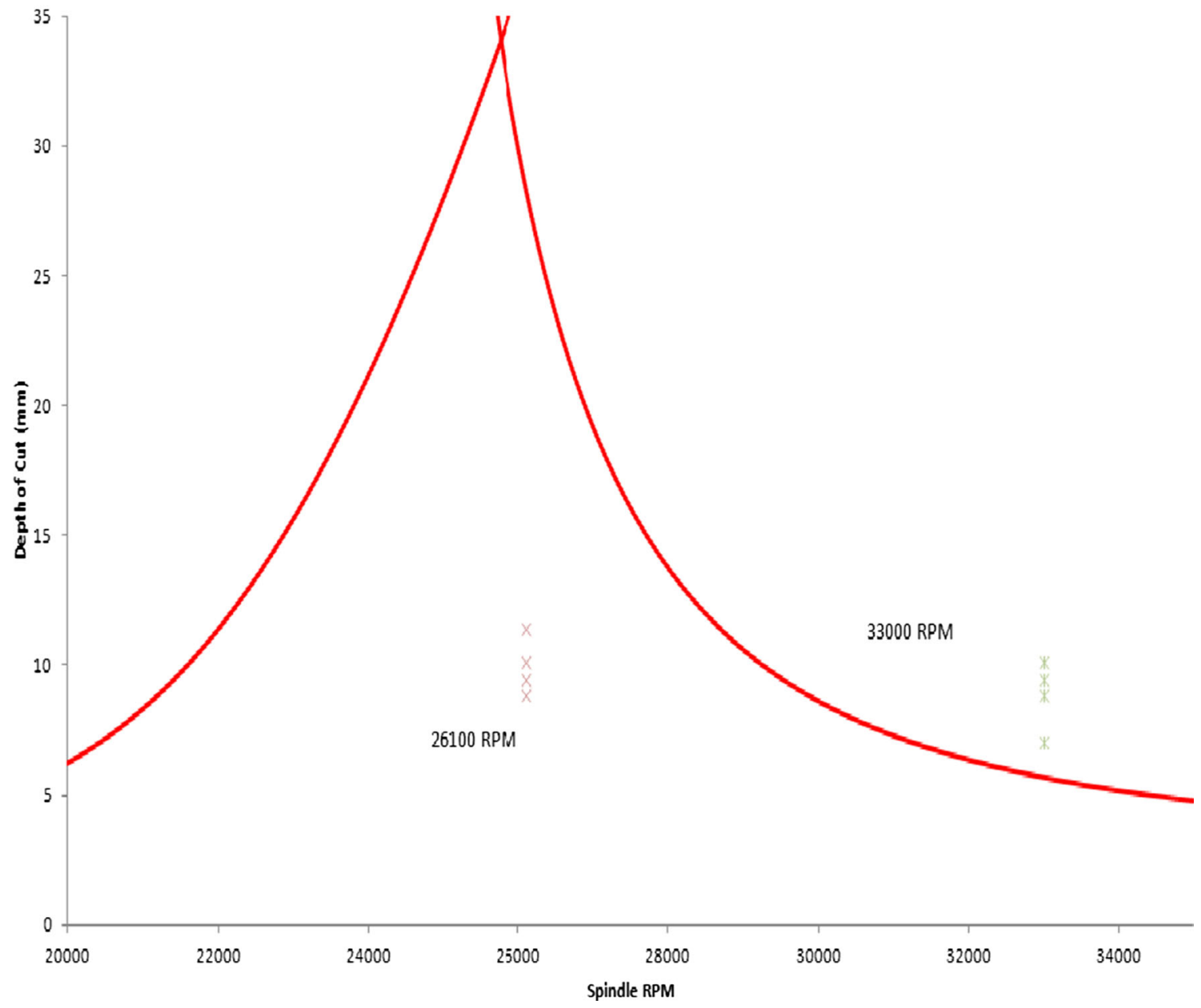

Fig. 17 Stability chart with test cuts (Merlin tool) zoomed in

Once the static stiffness matrix of the system, $[\boldsymbol{K}]$, has been evaluated, one can then apply any force to the node representing the tool tip in the spindle model and calculate the deflection, $U$, in the same direction as the applied force, $F$, using the following expression:

$[U]=[\boldsymbol{K}]^{-1}[\mathbf{F}]$

This deflection, $U$, is then used to calculate the theoretical tool stiffness, as follows:

$K_{\text {theo }}=\frac{F_{x}}{x}$

Now, consider a machining process (aluminium) with a full slot axial depth of cut, and using a two-tooth roughing tool. The following parameters were used to generate the stability lobes:
- System natural frequencies, $\omega_{n 1}=930 \mathrm{~Hz}$ and $\omega_{n 2}=$ $800 \mathrm{~Hz}$

- Equivalent system damping, $\zeta_{1}=0.032$ and $\zeta_{2}=0.036$; both the natural frequency and damping values were obtained from the FRF charts using the peak picking method described in [17]

- Calculated equivalent system cutting stiffness, $K_{\text {theo } 1}=$ $3.1359 \mathrm{e} 7 \mathrm{~N} / \mathrm{m}$, and $K_{\text {theo } 2}=3.0309 \mathrm{e} 7 \mathrm{~N} / \mathrm{m}$

- Tangential cutting constant for aluminium $K_{t}=8.00 \mathrm{e} 8 \mathrm{~N} /$ $\mathrm{m}[17]$

- Force angle $\beta=68^{\circ}$ [17].

Figure 11 shows that, as the natural frequency decreases, the stability lobes tend to shift to the left and downwards. The two natural frequency values were taken from Fig. 7. It is clear that, for the spindle with reduced frequency, the cutting parameters assumed at the beginning of the life cycle of the spindle will not generate chatter free cuts unless the shift in the lobes is taken into consideration. The optimal spindle speed for a $25-\mathrm{mm}$ depth of cut is approximately 27,000 
Table 3 Test cuts and resulting machine vibrational results

\begin{tabular}{lrrl}
\hline Tool supplier & DOC $(\mathrm{mm})$ & RPM & Tool vibration \\
\hline IMCO & 7.112 & 33,000 & 2.9 \\
& 7.620 & 33,000 & 3.1 \\
& 8.890 & 33,000 & 3.8 \\
& 9.525 & 33,000 & 4.1 \\
& 7.620 & 26,500 & 1.9 \\
& 8.890 & 26,500 & 1.9 \\
Helical & 9.525 & 26,500 & 2.1 \\
& 7.112 & 33,000 & 4.1 \\
& 7.620 & 33,000 & 4.4 \\
& 8.255 & 33,000 & 5.0 \\
& 8.890 & 33,000 & 5.5 \\
& 7.620 & 26,500 & 1.5 \\
& 8.890 & 26,500 & 2.3 \\
& 9.525 & 26,500 & 2.1 \\
& 7.112 & 33,000 & 2.4 \\
& 8.890 & 33,000 & 3.1 \\
& 9.525 & 33,000 & 5.6 \\
& 10.160 & 33,000 & 5.6 \\
& 8.890 & 26,100 & 3.0 \\
& 9.525 & 26,100 & 2.9 \\
& 10.160 & 26,100 & 3.2 \\
& 11.430 & 26,100 & 3.4 \\
\hline
\end{tabular}

rounds per minute (RPM) for the $930-\mathrm{Hz}$ spindle. However, as can be seen from Fig. 11, a spindle speed of 27,000 RPM will very likely generate chatter as the spindle ages to $800 \mathrm{~Hz}$. The optimal speed for the $800 \mathrm{~Hz}$ spindle is approximately 23 , 500 RPM. It is worth noting that, if an initial spindle speed of 24,500 RPM is selected, chatter would be controlled throughout the life of the spindle based on the stability lobe shift in Fig. 11.

\section{Stability lobe experimental results}

In order to validate our stability lobes calculation procedure, developed based on the method described in [17], a spindle equipped with a set of two cutting-edge tools, made by three different manufactures (IMCO, Helical and Merlin), was used and several test cuts were taken.

The spindle was tap tested prior to performing the test cut, and, for each cutting tool, the spindle's fundamental frequency, damping coefficient, and system's stiffness, $k$, were extracted from the FRF data (see Table 2), based on which stability lobes were generated (see Fig. 12, 13, 14, 15, 16 and Fig. 17); similar to the previous case (Fig. 11), the tangential cutting constant for aluminium, $K_{t}=8.00 \mathrm{e} 8 \mathrm{~N} / \mathrm{m}$, and force angle, $\beta=68^{\circ}$ [17], were used. The ideal spindle RPM was also determined. Full slot with varying axial depth of cuts were used at that RPM to perform the test cuts.

Test cuts were also performed at the spindle maximum allowable RPM as well as 33,000 RPM. The machine vibrational sensors were used to detect the chatter, i.e. excessive machine vibration. As can be seen from Table 3, when test cuts are taken outside the stable zone, higher vibration numbers are observed, which corroborate the stability lobe charts. An acceptable level of vibration, as per the spindle system manufacturer's instructions, is $2.5 \mathrm{~mm} / \mathrm{s}$.

\section{Conclusion}

A CDSM for the vibration analysis of machine tool spindles was presented and was shown to be an accurate technique to calculate the natural frequencies of production spindle systems. Based on the bearing stiffness values calculated from the CDSM models, the overall stiffness of spindle system, also called the theoretical tool stiffness, was evaluated, which, in turn, was used to generate the stability lobes for an illustrative production spindle example. It was found that the stability lobes would shift significantly over the life span of the spindle. If this shift is not taken into consideration, chatter-free machining will not be maintained throughout the life of the spindle. The presented procedure can be effectively used to predict the changes in the spindle system fundamental frequency over a period of time caused by ageing, i.e. reduced bearings stiffness. As a result, decreased machine down time resulting from frequent tap testing commonly practiced to evaluate system's actual fundamental frequency used to extract the stability lobes could be achieved.

Acknowledgments The authors wish to acknowledge the support provided by Natural Sciences and Engineering Research Council of Canada (NSERC) and Ryerson University.

Compliance with ethical standards • Disclosure of potential conflicts of interest

- Research involving human participants and/or animals

- Informed consent.

\section{References}

1. Ertürk A, Budak E, Özgüven HN (2007) Selection of design and operational parameters in spindle-holder-tool assemblies for maximum chatter stability by using a new analytical model. Int J Mach Tools Manuf 47(9):1401-1409

2. Altintas Y, Cao Y (2004) A general method for the modeling of spindle-bearing systems. J Mech Des 126(6):1089-1104

3. Lin CW, Lin YK, Chu CH (2013) Dynamic models and design of spindle-bearing systems of machine tool: a review. Int J Precis Eng Manuf 14:513-521 
4. Guo Y, Parker R (2012) Stiffness matrix calculation of rolling element bearings using a finite element/contact mechanics model. Mech Mach Theory 51:32-45

5. Zhu L, Zhu C, Yu T, Shi J, Wang W (2007) Dynamic analysis and design of the spindle-bearing system in Turn Milling Centre, Int Conf Autom Logistics. 2007 IEEE

6. Cao Y, Altintas Y (2007) Modeling of spindle-bearing and machine tool systems for virtual simulation of milling operation. Int J Mach Tools Manuf 47:1342-1350. doi:10.1016/j.ijmachtools.2006.08.006

7. Hajikolaei K, Moradi H, Vossoughi G, Movahhedy M (2010) Spindle speed variation and adaptive force regulation to suppress regenerative chatter in the turning process. J Manuf Process 12:106-115

8. Graham E, Mehrpouya M, Park SS (2013) Robust prediction of chatter stability in milling based on the analytical chatter stability. J Manuf Process 15:508-517

9. Qin, F. Gong, X. Chou, K (2011) Size effect in cutting with diamondcoated tools. ASME 2011 International Manufacturing Science and Engineering Conference, June 13-17, 2011, pp. 267-273

10. Banerjee JR, Su H (2004) Free vibration analysis of a spinning composite beam using the dynamic stiffness method, Scotland. Proceedings of the Seventh International Conferences on Computational Structures Technology, B.H.V. Topping and C.A. Mota Soares. Civil-Comp Press
11. Gaber O, Hashemi SM (2014) Research article on the free vibration modeling of spindle system: a calibrated dynamic stiffness matrix. Shock Vibr 2014 Article ID 787518, 10 pages

12. Blevins R (2001) Formulas for natural frequency and mode shapes. Krieger Pub Co, USA

13. Sada, Y (2012) FE modal analysis of a non-spinning machine tool spindle. Internal Report, Department of Aerospace Eng., Ryerson University, Toronto, Canada

14. Marciniak M (2012) Modal analysis of a tool holder system for a five-axis milling machine. 2012, B.Eng. Thesis, Department of Aerospace Eng., Ryerson University, Toronto, Canada

15. Banerjee JR (1997) Dynamic stiffness formulation for structural elements: a general approach. Comput Struct 63(1):101103

16. Hashemi SM (1998) Free vibrational analysis of rotating beam-like structures: a dynamic finite element approach. PhD. thesis, Department of Mechanical Engineering, Laval University, Québec (QC), Canada, September 1998.

17. Schmitz T, Smith K (2009) Machining dynamics. Springer, New York

18. Richards TH, Leung YT (1977) An accurate method in structural vibration analysis. J Sound Vib 55(3):363-376 